\title{
UNDERSTANDING LARGE SOFTWARE SYSTEMS WITH THE UTILITY XREF
}

Clifford E. Rhoades, Jr.

UCRL-ID--106165

DE91 007630

\begin{abstract}
When a software system reaches a certain size, arteriosclerosis sets in. That is, the system becomes harder and harder to add new features and even more difficult to understand. Worse, the insertion of new features often introduces new programming errors as well as revealing ones already present but previously unmanifested. For Fortran programs, the 100,000 line size is critical. XREF fights hardening of the arteries by providing the user information on the organization, variable usage, and common block use within the software system.

XREF is language independent. That is, it works, for example, with CAL, C, CIVIC, and CFT complied subprograms. XREF performs its global symbol analysis from either the BUILD library file or the object (binary) file. Naturally more useful information is obtained when a symbol table is generated by the compiler. Specifically, eight global reports are produced. The most helpful report consists of a listing of all symbols contained in the BUILD library or binary file, the subprograms that use the symbol and the relocation basis (either local or common block name) of each symbol. Five secondary reports are provided for each subprogram.
\end{abstract}

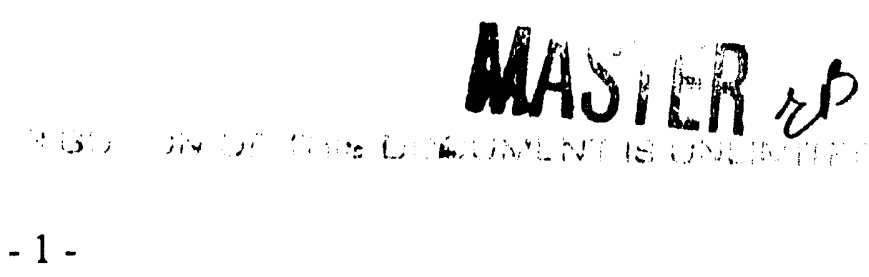


CONTENTS

TITLE

PAGE

$\begin{array}{ll}\text { ABSTRACT } & 1\end{array}$

CONTENTS 2

INTRODUCTION 3

GENERAL DESCRIPTION 3

RESTRICTIONS

OPERATION 5

EXAMPLE OF OUTPUT

ERROR MESSAGES 5

REVISIONS

REFERENCES

$\begin{array}{ll}\text { APPENDIX I } & 7\end{array}$

APPENDIX II $\quad 24$ 


\section{UNDERSTANDING LARGE SOFTWARE SYSTEMS WITH THE UTILITY XREF}

\section{Clifford E. Rhoades, Jr.}

\section{INTRODUCTION}

As most people who work on large software systerns have experienced, as a software system gets larger and larger, it becomes more and more difficult to understand. Most of the life cycle cost of a large program is not in its development. Rather, it is maintenance that really makes software expensive. Maintenance is often a code work for "changes in external specification" and not just repair of errors or changes to accommodate new operating environments. One more problem with any long term software effort is the constant turn over in people. At Livermore, we have software efforts going on whose originators have long since retired. Turnover in programming staff seems to be faster than turnover in physicists, but nevertheless changes in personnel on any project are inevitable.

XREF was written to aid new and old staff in the never ending battle to understand what has been programmed and how new features can be added. It produces a set of useful reports based on the contents of the binary (object) file or the BUILD library of a program. More details are contained in the following section.

\section{GENERAL DESCRIPTION}

XREF performs a global code analysis of BUILD library files or binary files consisting of routines complied by CIVIC, CFT, CFT77, or any other compiler which generates standard (UNICOS) symbol tables. XREF is patterned on BINED written by Terry H. Gong for the 7600. XREF produces 8 reports on the primary output file and a subprogram by subprogram analysis on the secondary output file.

The eight reports are:

1. a sorted list of subprogram names and various identification information;

2. the entry points contained in each subprogram;

3. the subprogram in which each entry is found;

4. the common blocks used by each subprogram;

5. a list of subprograms which use each common block;

6. a list of externals called by each subprogram;

7. a list of subprograms which call each subprogram; and finally,

8. a list of subprograms which use each symbol.

This last report consists of a listing of all symbols contained in the BUILD library or binary file, the subprograms that use the symbol and whether the symbol is local to the subprogram or the 
name of the common block in which the symbol is contained.

The primary output is written to an 8-bit ASCII print file whose name is formed by the concatenation of $\mathrm{H}$ and the current suffix with the name of the input file truncated to 6 characters, if necessary. The primary output file contains a header consisting of the name of the binary file, the machine on which XREF was run, the suffix under which it was run, and the date and time when XREF was run. After the above mentioned eight reports, the primary output listing is completed with the XREF execution time in seconds including the CPU, IO, system and total times.

The secondary report contains the following information for each subprogram:

1. subprogram name

2. entry points

3. exiernal references

4. local variables used

5. variables used in each common block.

The secondary output file is written to an 8-bit ASCII print file whose name is formed by the concatenation of $\mathrm{P}$ and the current suffix with the first six characters of the input file name. After the above mentioned subprogram by subprogram breakdown, the secondary output listing is completed with the XREF execution time in seconds including the CPU, I/O, system, and total times. Note that currently NLTSS provides a zero rather than the correct system time.

\section{RESTRICTIONS}

XREF analyzes only one binary file or BUILD library file at a time. For maximum usefulness, the individual subprogram modules within the binary or library file should contain a symbol table.

The current version can handle as many subprogram names, entry point names, common block names, external references, local variables and common variables as will fit into memory on the computer on which XREF is run. The exact amount depends on the maximum field length allowed, the garbage collection scheme used by the supporting routines, and the order in which nominal initial sizes are exceeded.

Currently XREF only used the first eight letters of symbol names. This could be extended easily to 16 or even 24 at the price of more memory requirements and much greater complexity of formatting the print outs.

When the compiler sets the necessary flags, XREF indicates the usage of a variable in a subprogram. That is, it distinguishes between variables referenced and variables defined. Variables defined are indicated by an $=$ sign before the subprograms in which the variables are set. Note that at the present time not all compilers set the flags appropriately. CIVIC appears to still be the best in this regard.

The primary and secondary output file lengths are limited to 512,000 decimal words but 
may be extended in the operating system. There are no help facilities or interactive commands. Standard FORTLIB error messages and those given below are provided.

\section{OPERATION}

The controllee for XREF can be obtained from mass storage via:

$$
\text { XP READ .743150:XREF }
$$

The execute line is

XREF binary_file_name

where binary_file_name is the name of BUILD library or binary (object) file consisting of eight or fewer characters.

If binary_file_name is omitted, a request for it will be made. The primary output file is named by concatenating $\mathrm{H}$ (for high speed print file), with the current suffix, and the first 6 characters of the binary file name. The secondary output file is named by concatenating $P$ (for print file), with the current suffix and the binary file name truncated to 6 characters, if necessary. The drop file name is +XREFy, where $y$ is the current suffix.

For example, the execute line

\section{XREF CLIFF}

typed under suffix A will produce the primary output file HACLIFF, the secondary output file PACLIFF, and the drop file +XREFA.

\section{EXAMPLES OF OUTPUT}

The two appendices give examples of running XREF on the binary produced by compiling XREF. Appendix I is the primary output file, while Appendix II contains the secondary output file. Lines with the number " 1 " in column 1 begin a new page.

\section{ERROR MESSAGES}

The following table of error messages and explanations is provided to aid the user.

Too many input symbols

Too many letters in file name

Input file does rot exist

Cannot create output file

\section{Explanation}

Too many file names were specified on the execute line or in response to the request for the input binary tile name.

Input file name is restricted to eight or fever characters.

An open attempt on the input file returned a length file size. Restart XREF with the correctly spelled input file name.

There is insufficient disk space to create an output file. 


\section{REVISIONS}

The original XREF program was written in late summer 1981 and installed September 1, 1981. It was revised in 1986 because the old FORTLIB memory management routines were deleted and a new scheme was adopted. Both the 1988 and the 1990 revisions were made necessary by changes in symbol table formats.

\section{REFERENCES}

1. Terry Gong, BINED, UR-111 (June 3, 1971).

2. Paul Thompson, SYMEDIT, Lawrence Livermore National Laboratory, Livermore, CA 94550, UCIR-1339 (February 4, 1980).

3. Cray Research, Inc., Deb'ıg Tables Internal Reference Manual, SM-0225D (1990). 
APPENDIX I

$-7-$ 
$>$

$\frac{E}{U}$

$\stackrel{\circ}{\circ}$

$\stackrel{\infty}{*}$

5

$\underset{+\infty}{\mathbb{N}}$

0
$a$
1
2
1
5
-
0
0
0

$\infty$
4
4
2
$\frac{1}{2}$

1

,

(1)

$-$

in

4

$-8-$ 


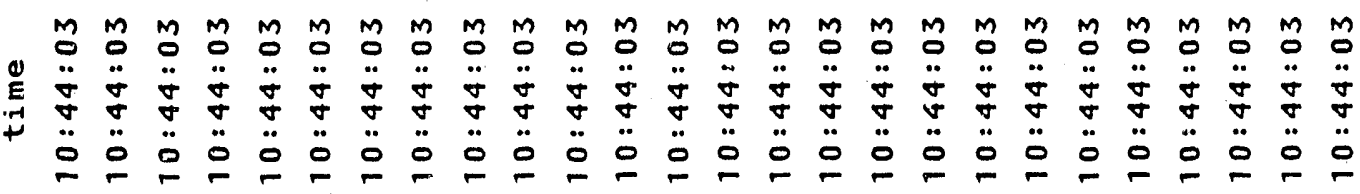

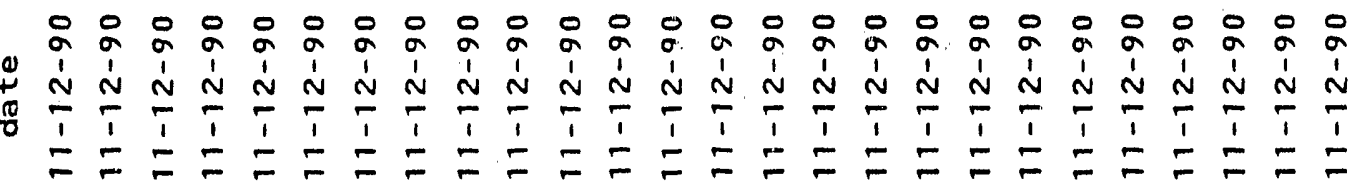

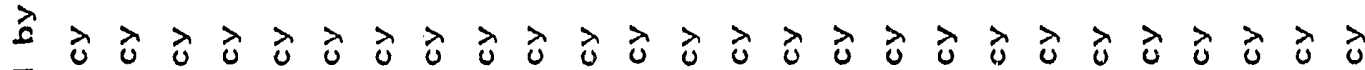
d

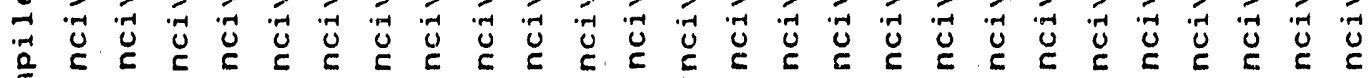

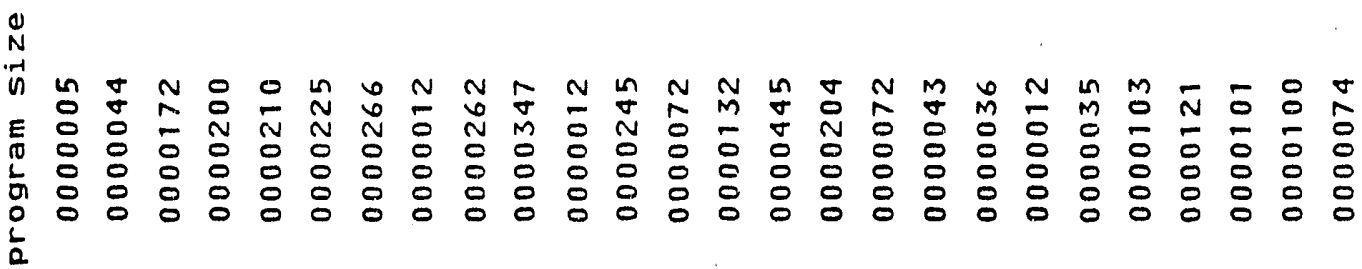

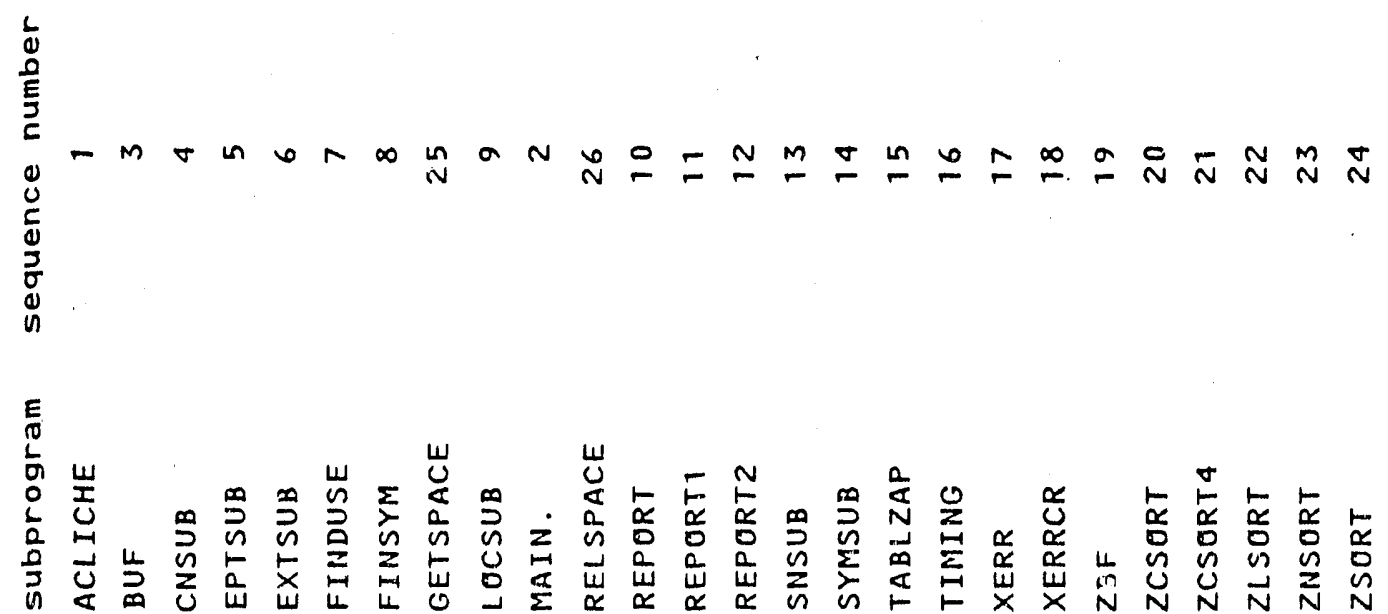




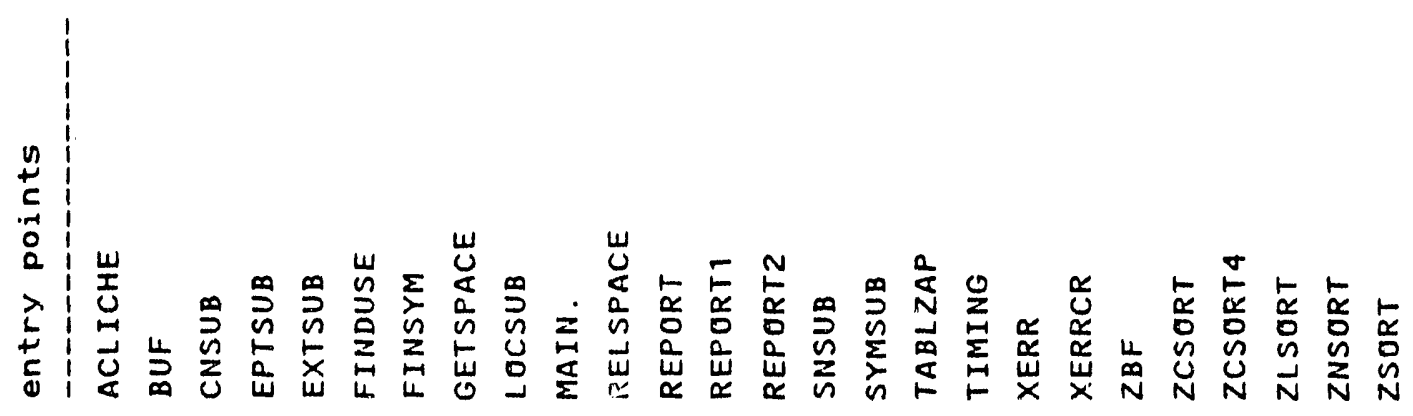

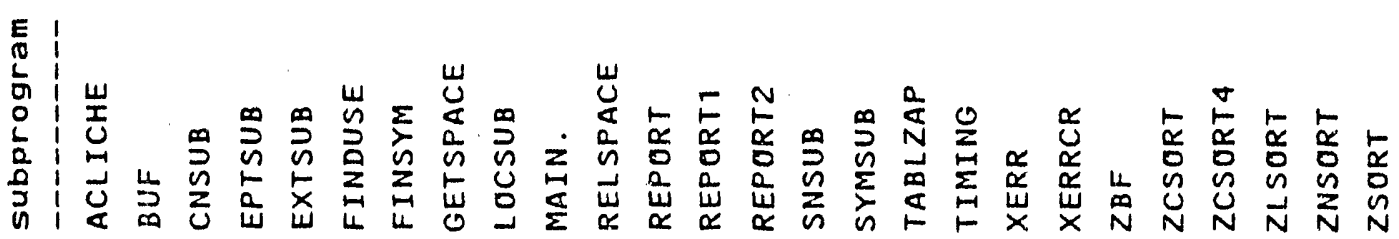




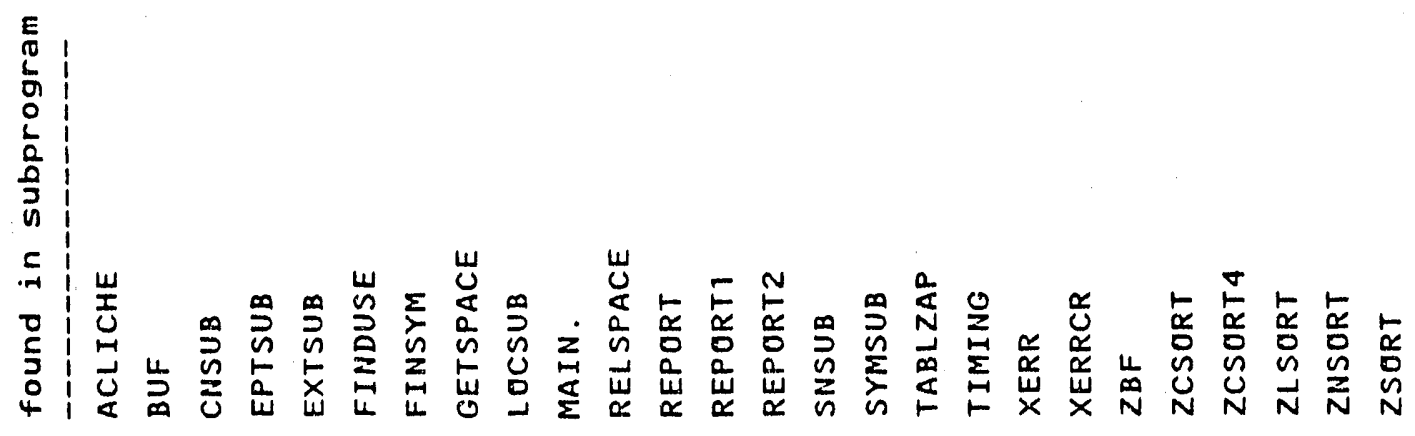

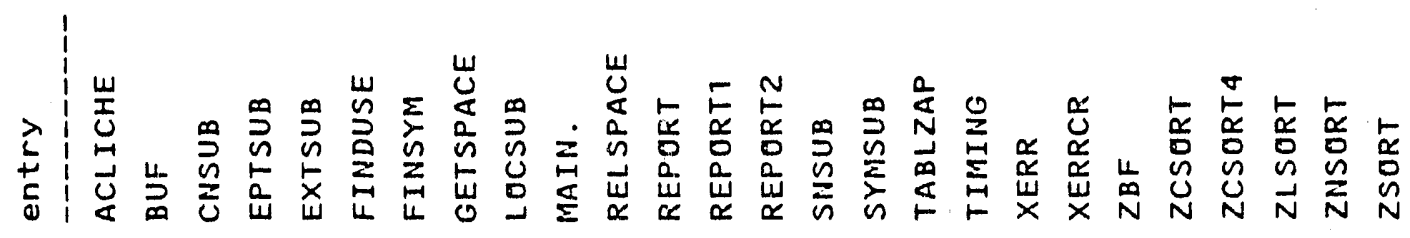


$\frac{\overrightarrow{1}}{n}$

$\frac{0}{\substack{\alpha \\ a}}$

능

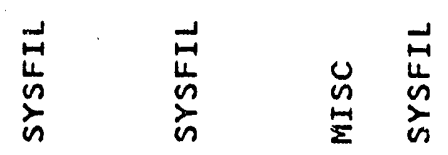

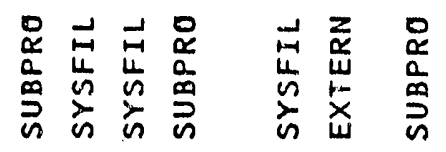

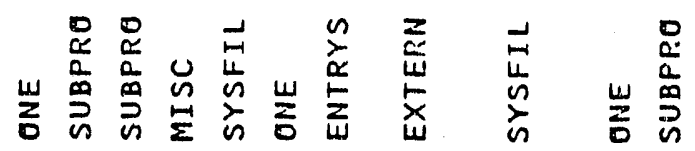

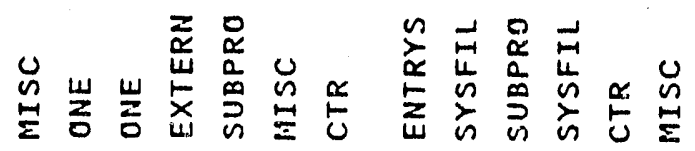

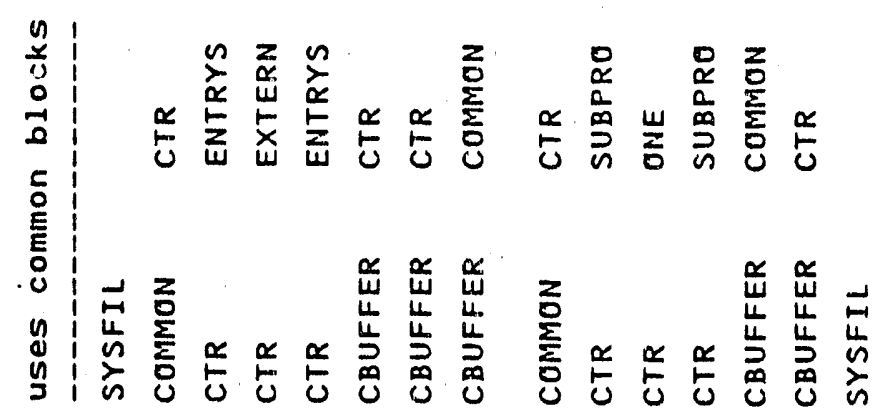

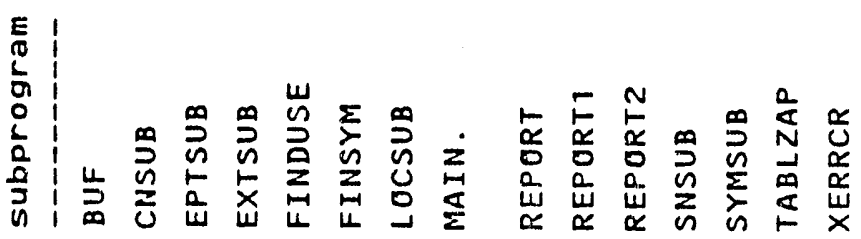




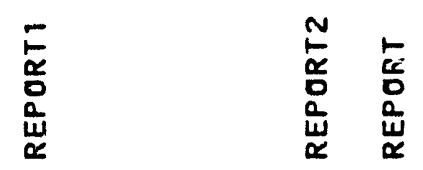

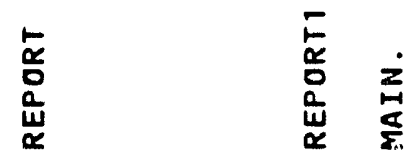

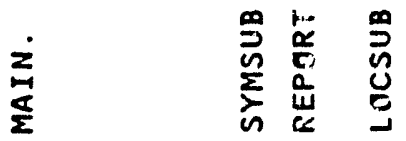

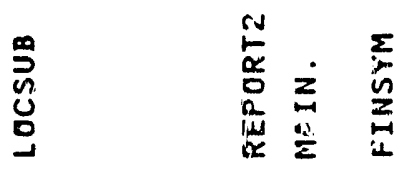

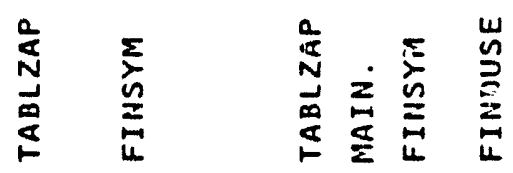

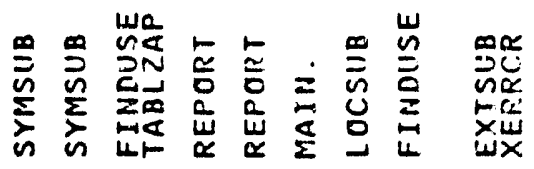

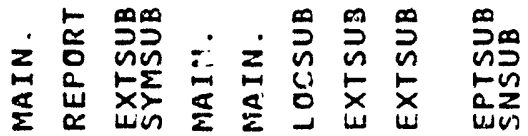

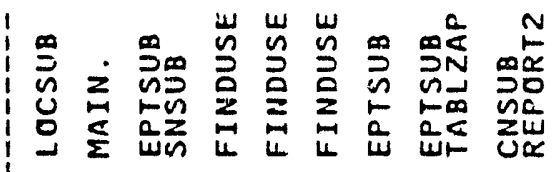

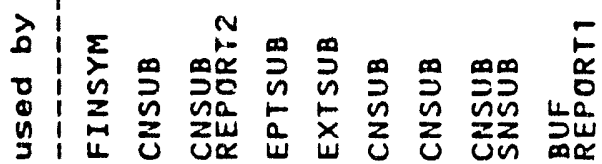

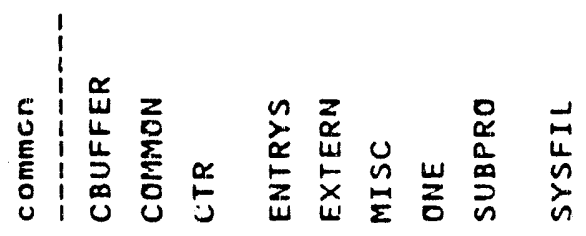




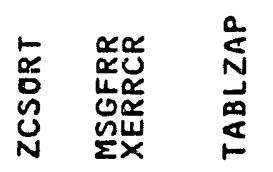

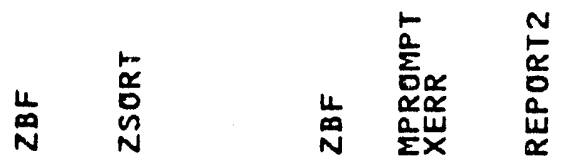

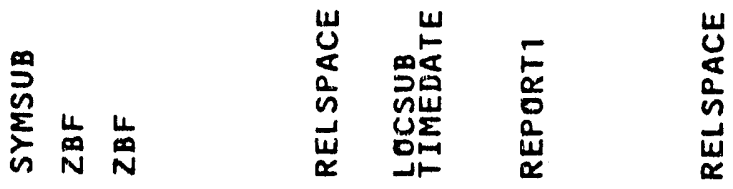

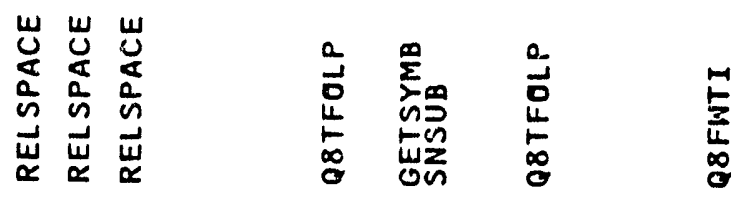

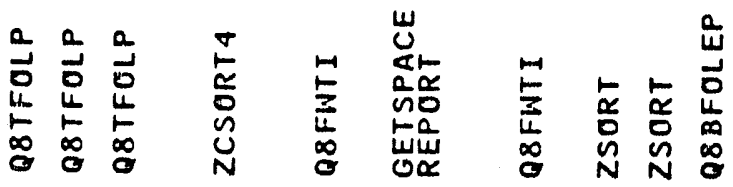

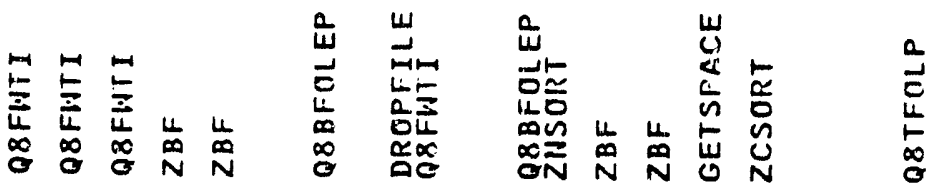

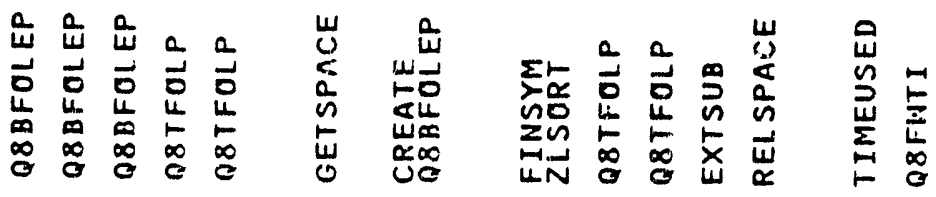

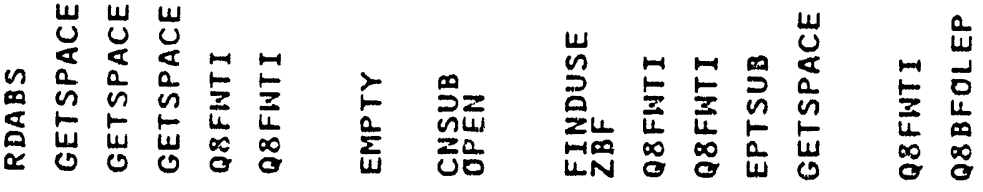

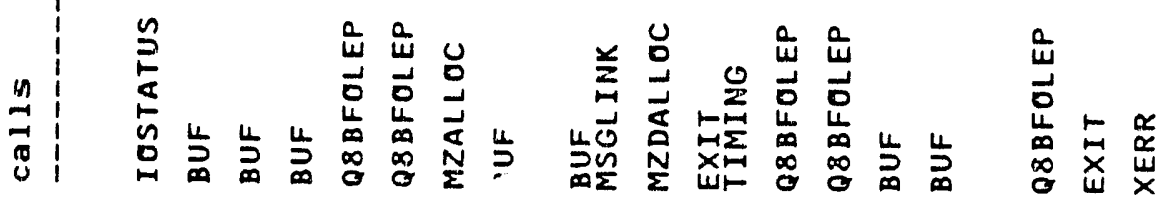

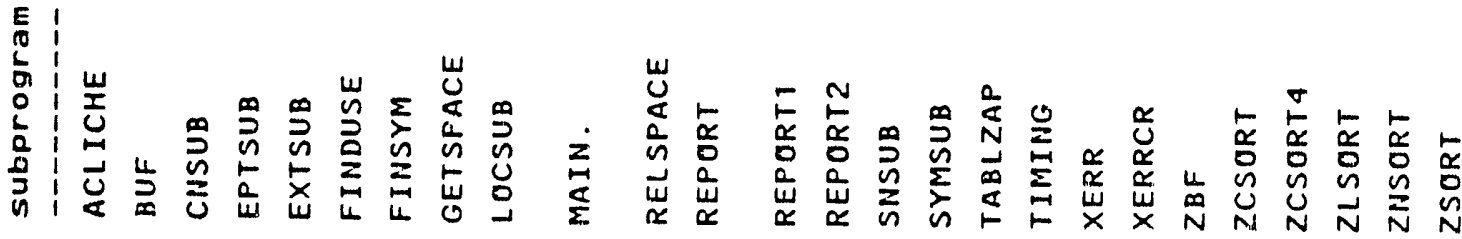


In

经

1

in

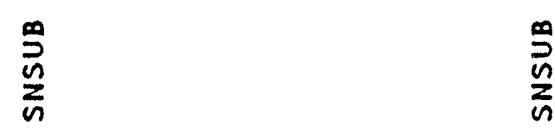

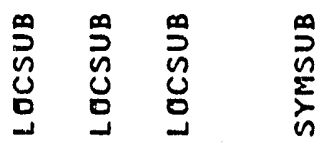

$\sum$

$\stackrel{\Sigma}{E-1}$

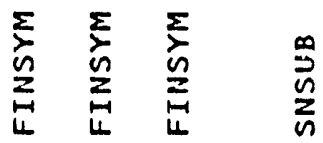

$\sqrt{1}$

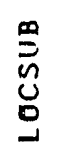

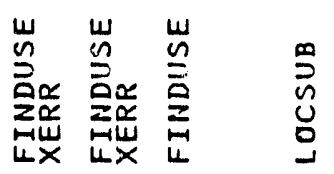

敛

号

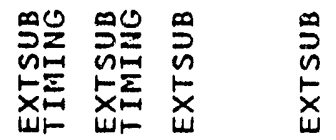

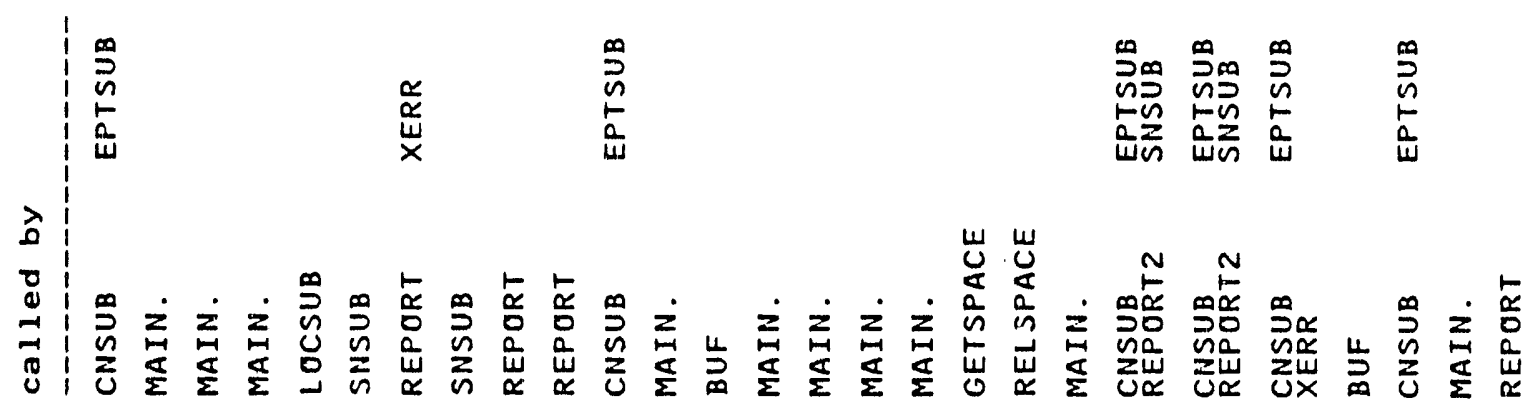

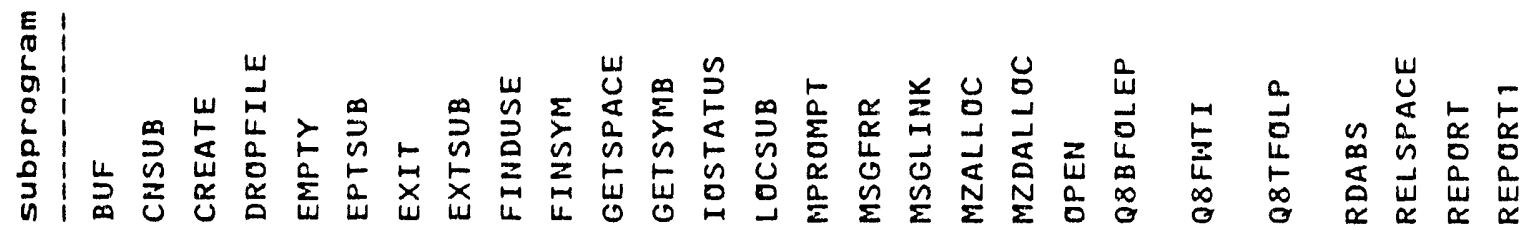


$\stackrel{N}{\circ}$
$\frac{0}{0}$
$\frac{0}{\alpha}$
$\frac{1}{\alpha}$

$E$
$\frac{0}{0}$
$\frac{1}{w}$
$\frac{1}{\alpha}$

$\leftarrow$
$\frac{\alpha}{0}$
$\frac{1}{\sim}$
$\propto$

$\stackrel{\infty}{0}$

$\sum_{\substack{n \\ n}}^{n}$

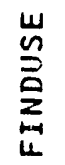

$\stackrel{\infty}{\stackrel{n}{5}}$

$N$
$\frac{N}{0}$
$\frac{\alpha}{w}$
$\frac{0}{\alpha}$

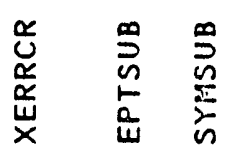

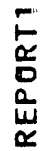

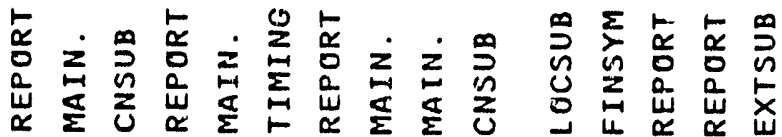

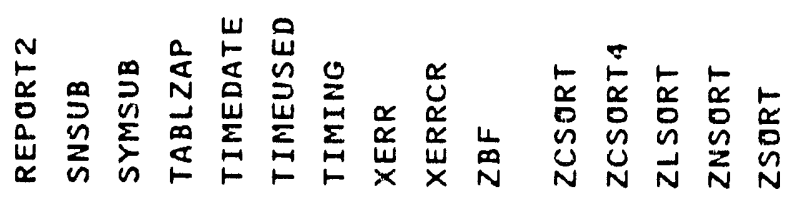




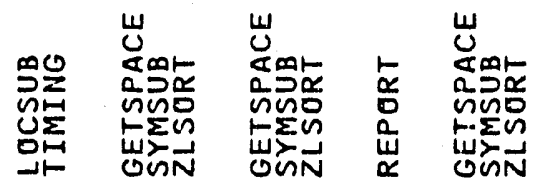

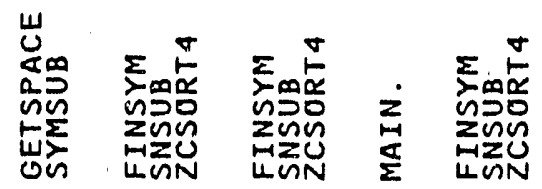

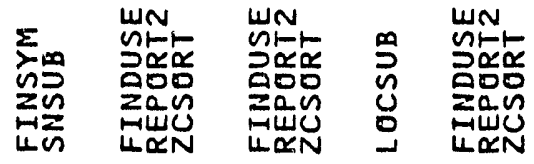

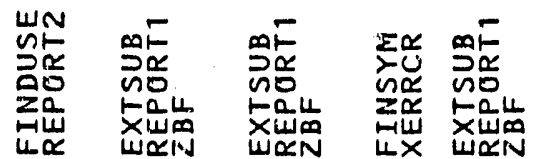

\&
ơ
$N$

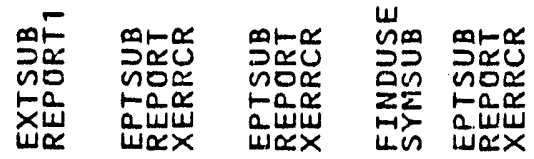

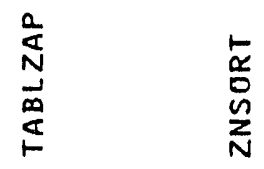

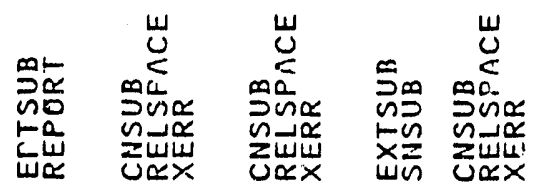

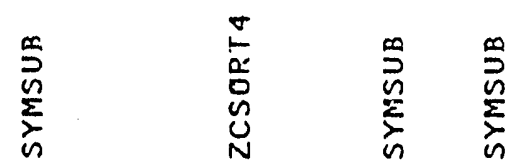

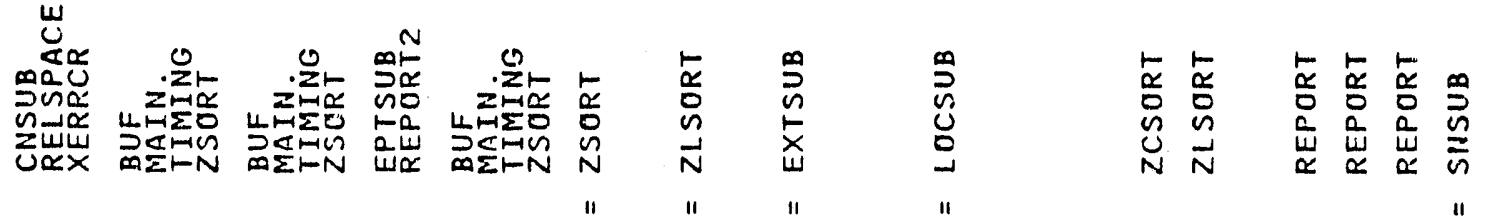

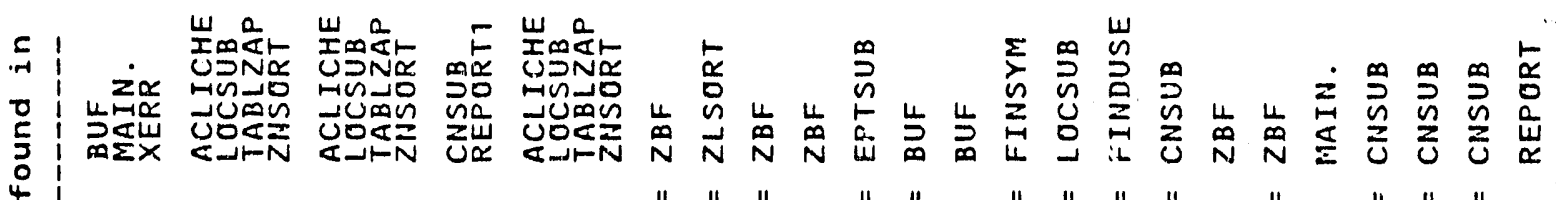

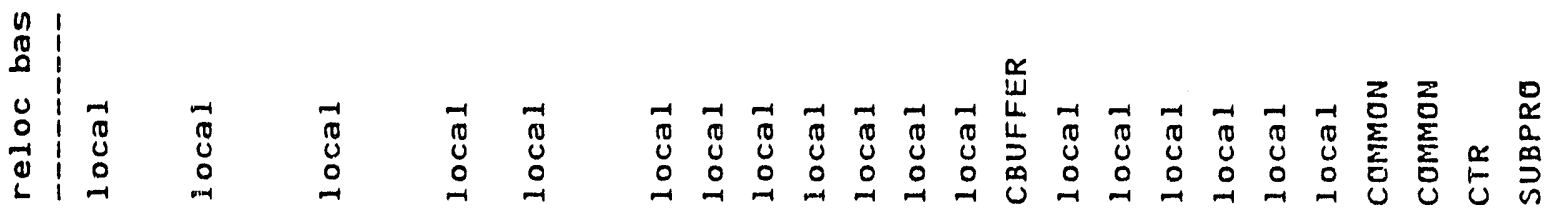

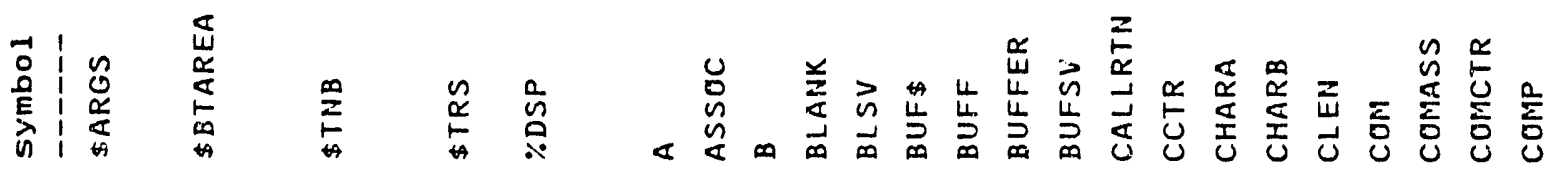




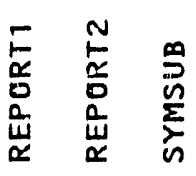

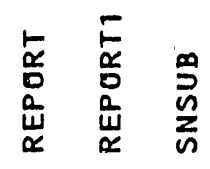

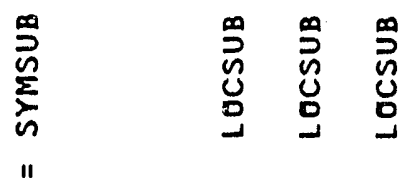

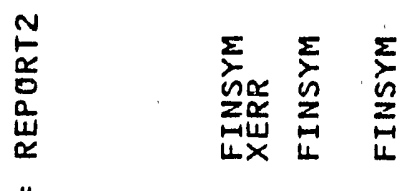

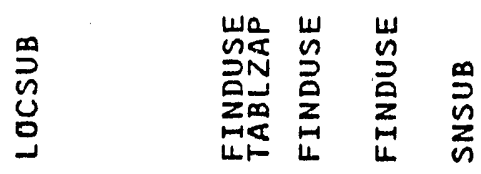

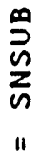

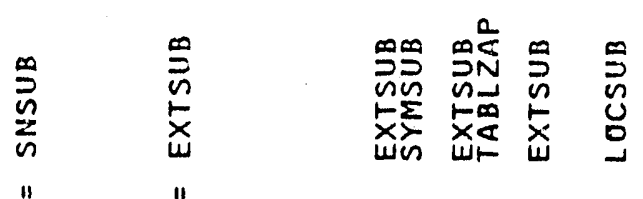

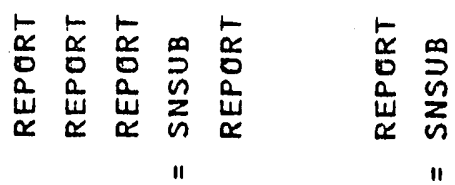

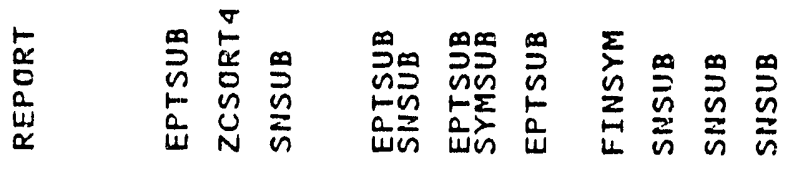

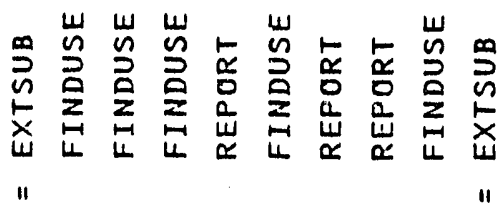

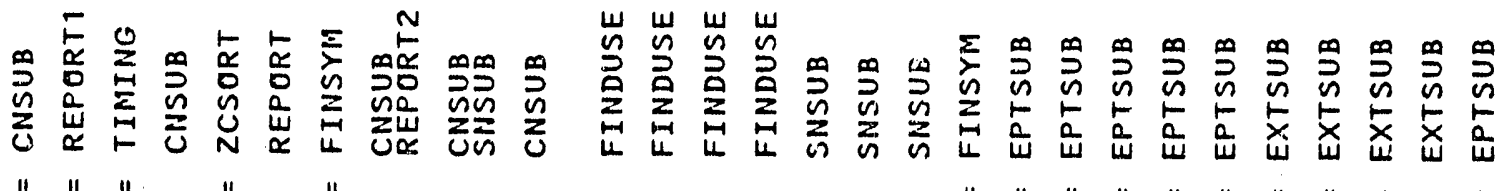

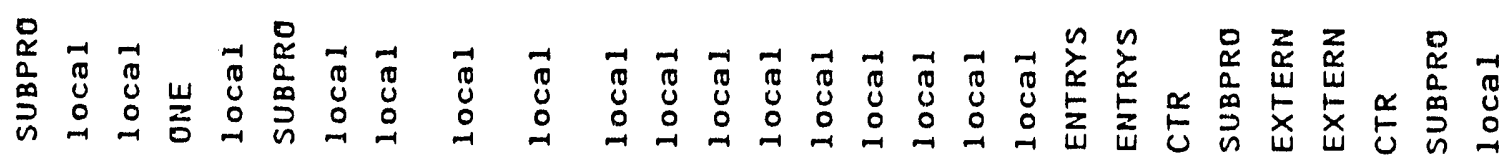

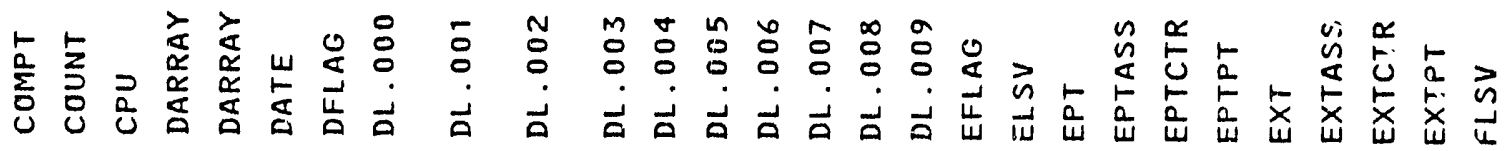




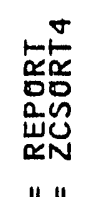

囍高喜

"I II

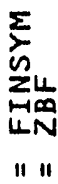

$\sum_{n}^{\infty}$

แ

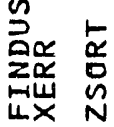

$\underset{⿱ 亠 䒑}{\Sigma}$

" " "

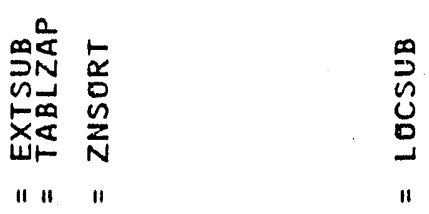
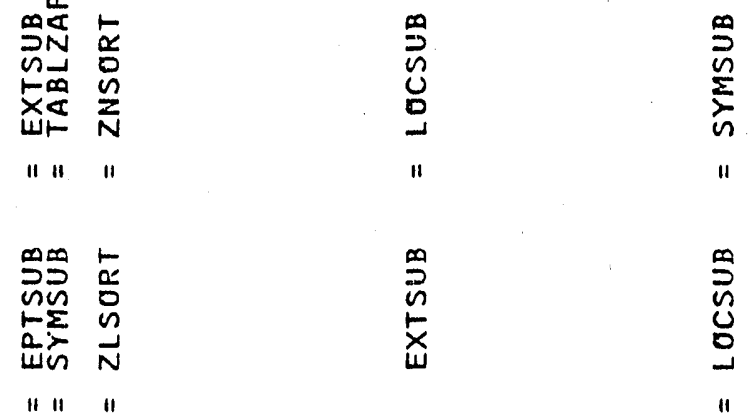

" I"
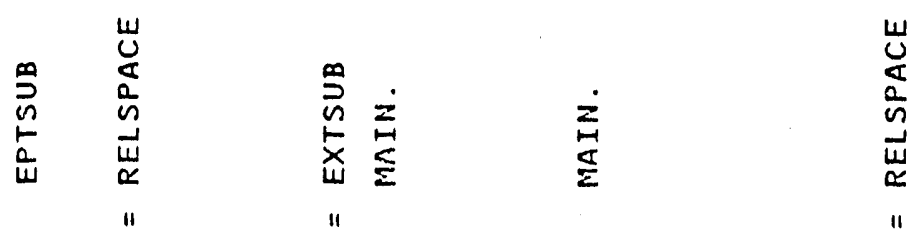

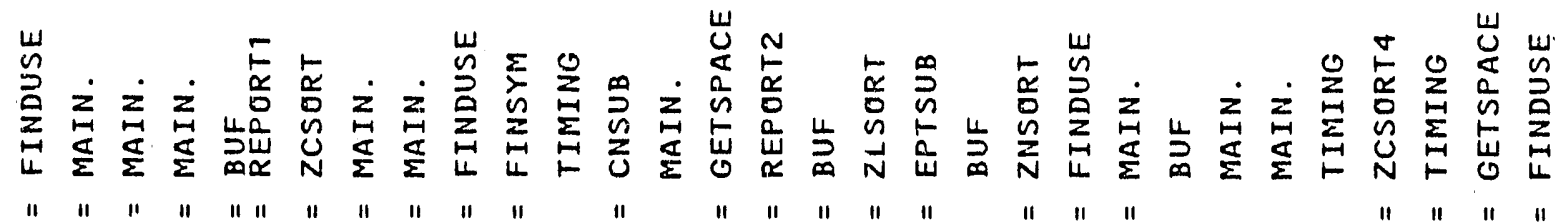

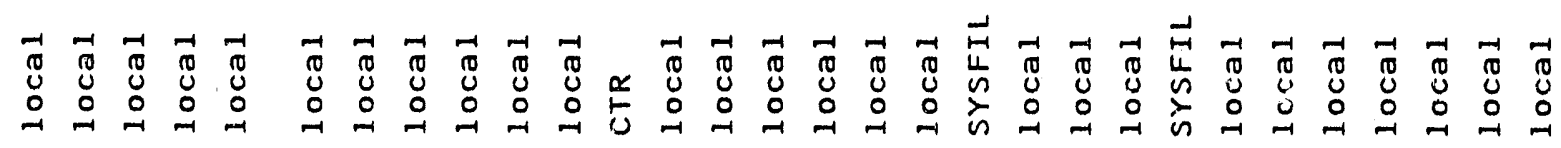

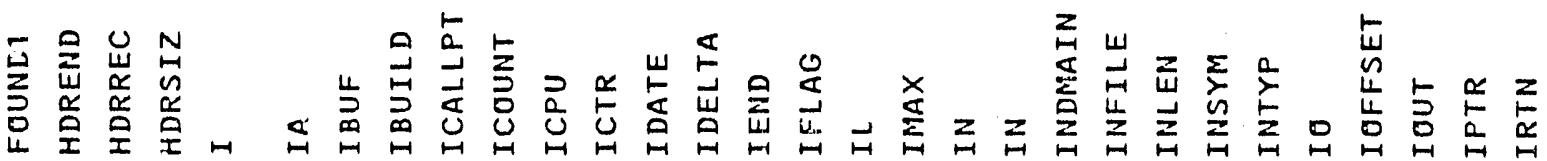



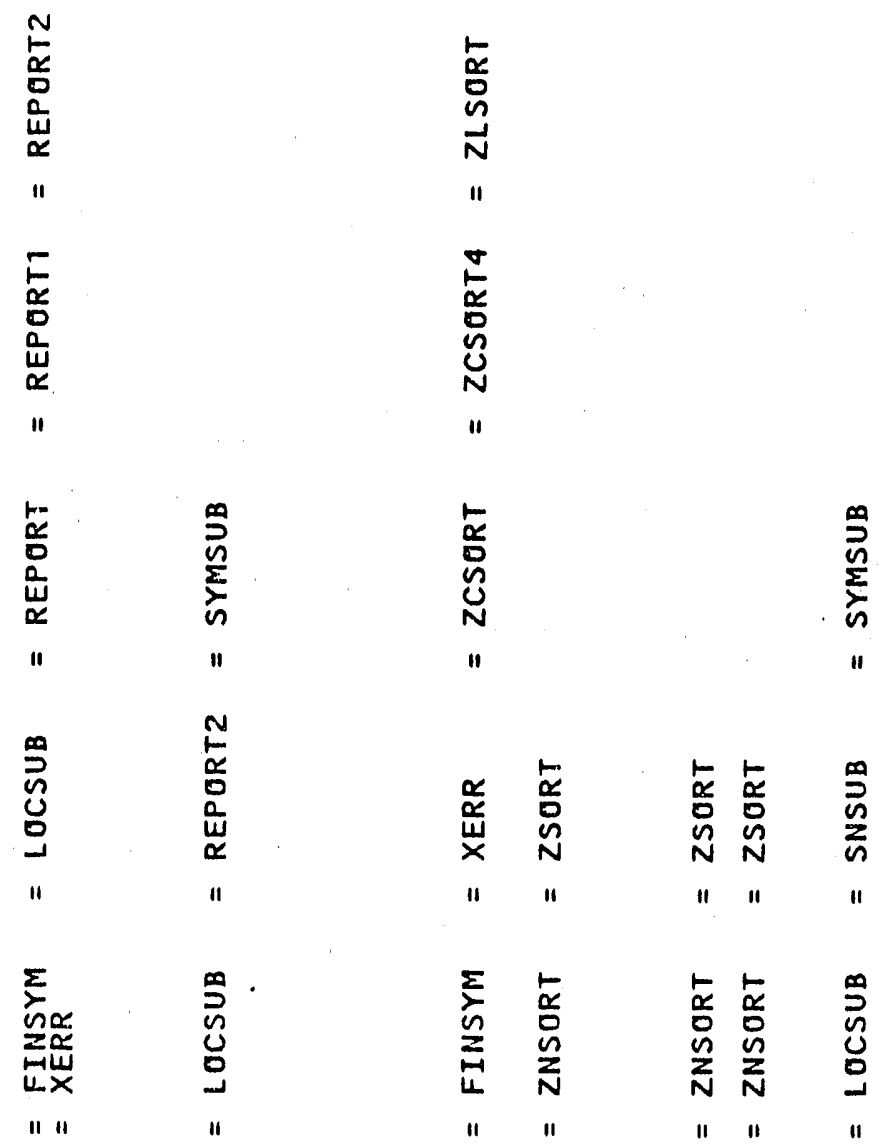

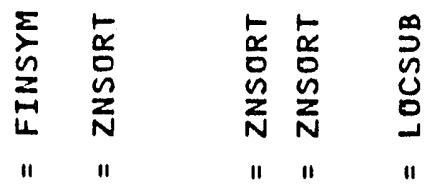

\begin{tabular}{|c|c|c|c|c|c|c|}
\hline 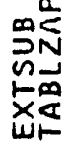 & $\begin{array}{l}\stackrel{\infty}{\partial} \\
\tilde{\omega} \\
\stackrel{5}{x}\end{array}$ & 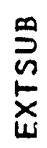 & $\begin{array}{l}5 \\
0 \\
0 \\
\sim \\
\vec{N}\end{array}$ & $\begin{array}{l}\frac{\sigma}{0} \\
0 \\
\sim \\
N\end{array}$ & $\begin{array}{l}\frac{5}{\alpha} \\
\stackrel{\Delta}{N} \\
\vec{N}\end{array}$ & $\sum_{\substack{n \\
n}}^{\infty}$ \\
\hline " & "I & 11 & "1 & $\|$ & $"$ & "1 \\
\hline
\end{tabular}

\begin{tabular}{|c|c|c|c|c|c|c|c|c|}
\hline 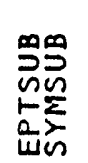 & $\begin{array}{l}\dot{\sigma} \\
\stackrel{\alpha}{0} \\
\tilde{U} \\
\tilde{N}\end{array}$ & $\begin{array}{l}\stackrel{n}{2} \\
\stackrel{n}{5} \\
\frac{0}{n}\end{array}$ & $\begin{array}{l}\frac{5}{\alpha} \\
0 \\
N \\
N\end{array}$ & 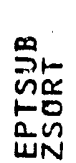 & $\begin{array}{l}\tilde{E} \\
\frac{\alpha}{0} \\
\tilde{N}\end{array}$ & $\begin{array}{l}E \\
E \\
0 \\
0 \\
0 \\
N\end{array}$ & 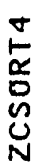 & $\underset{i=1}{\sum}$ \\
\hline u & " & $\|$ & 11 & " " & II & " & " & 11 \\
\hline
\end{tabular}

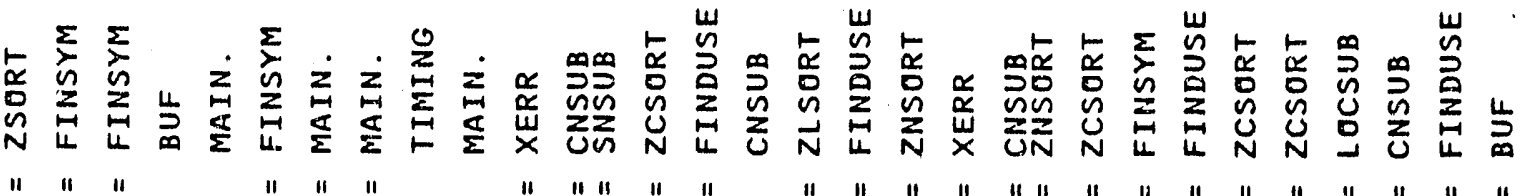

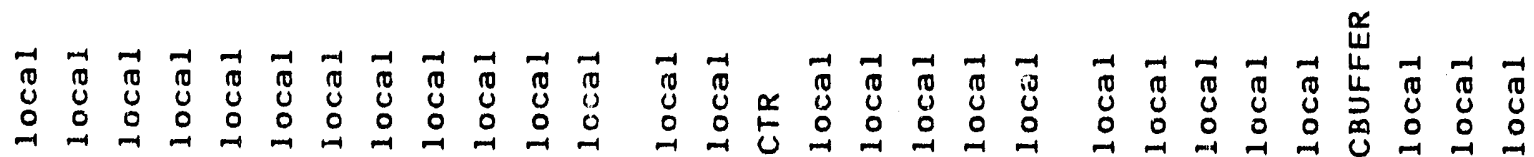

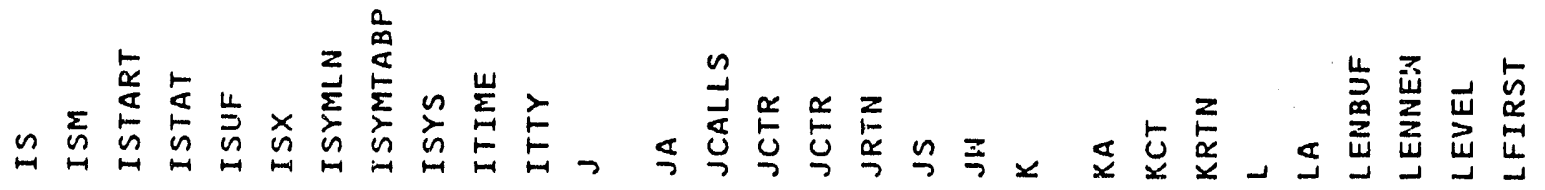




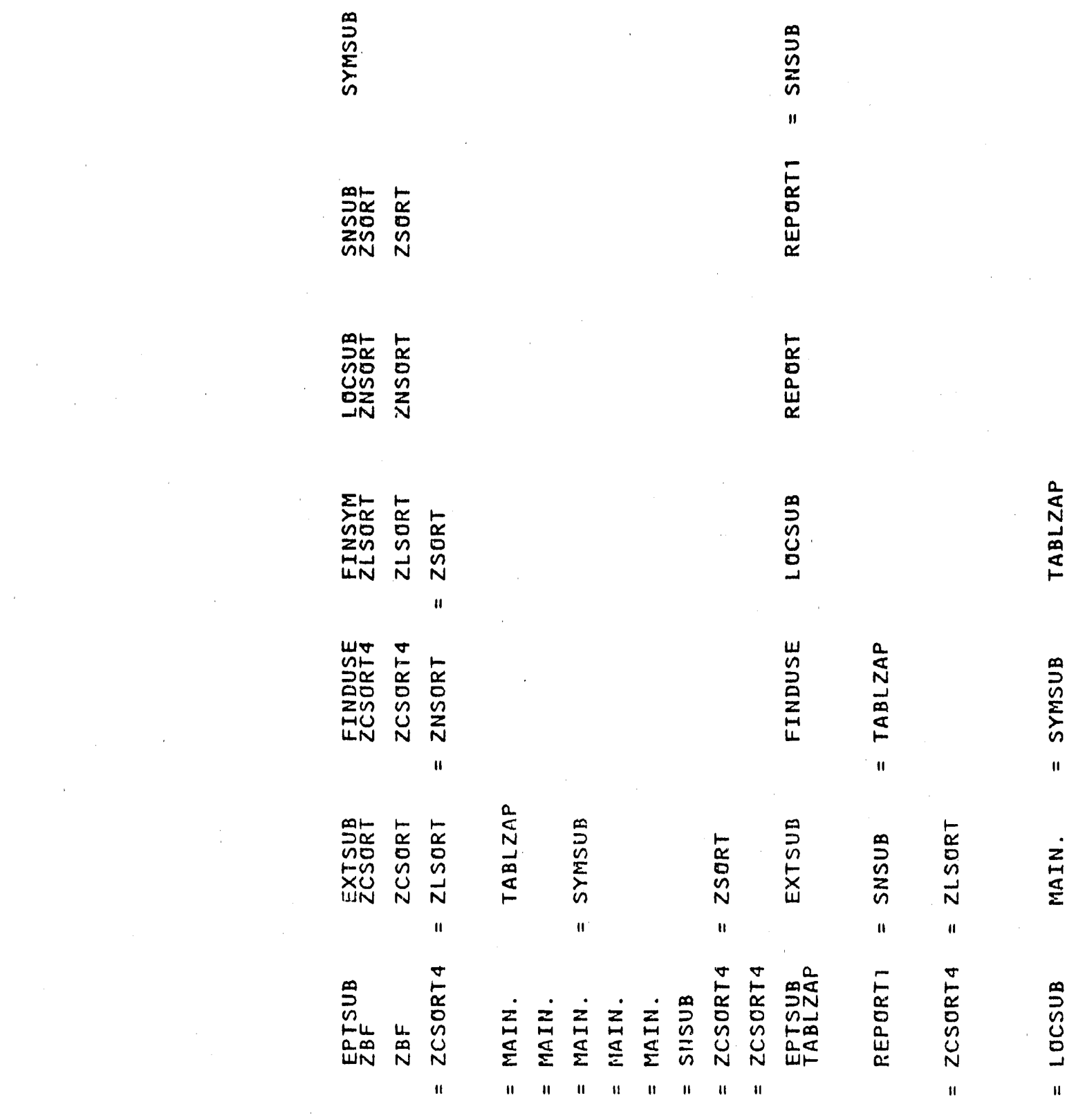

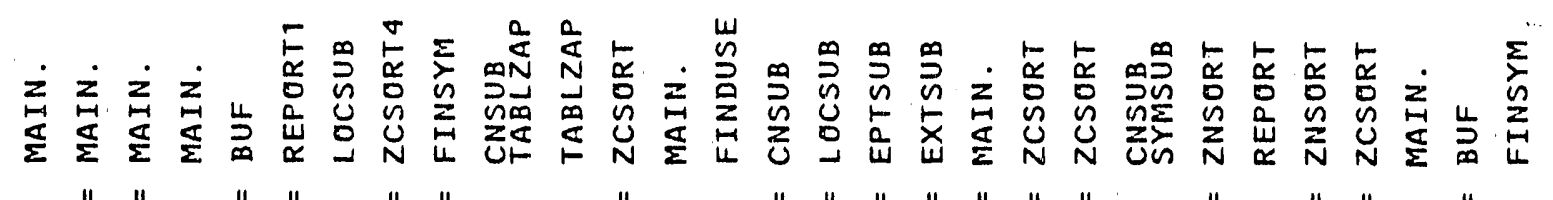

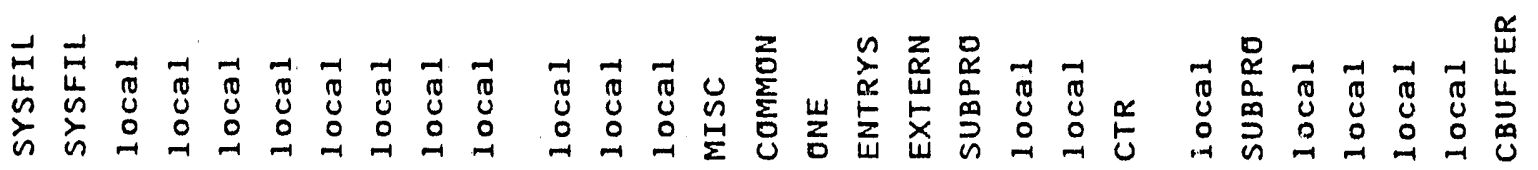

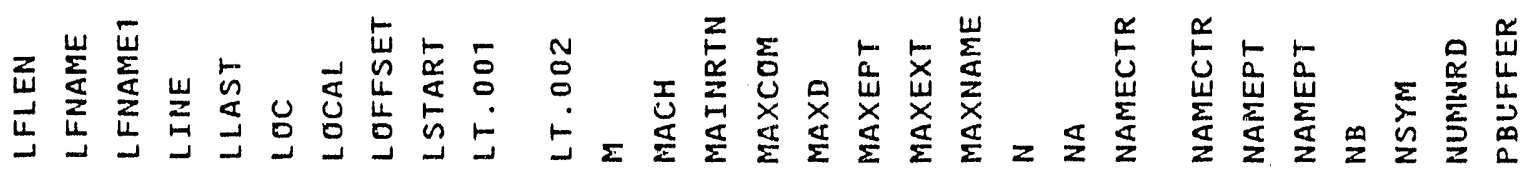




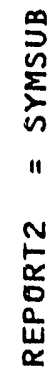

$\sum_{n}^{\infty}$

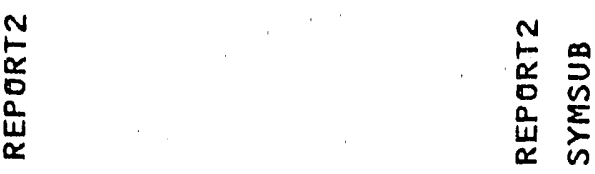

$\sum_{\substack{n \\ n}}^{\infty}$

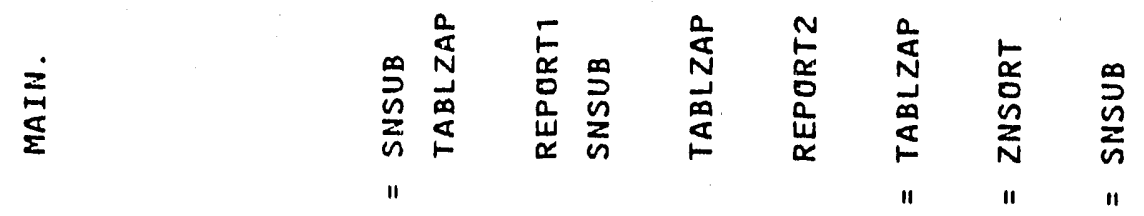

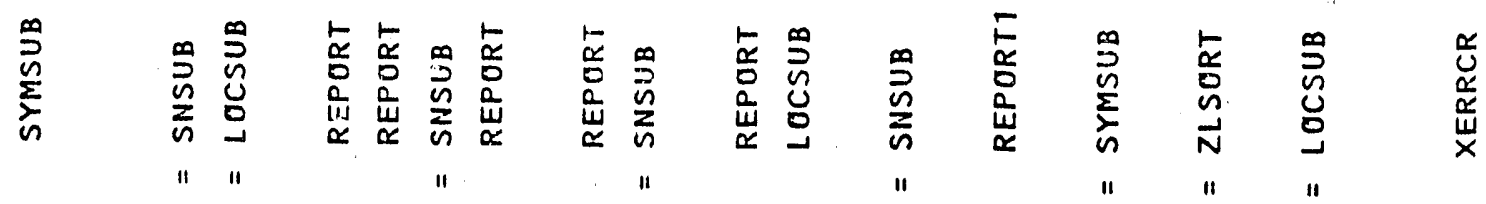

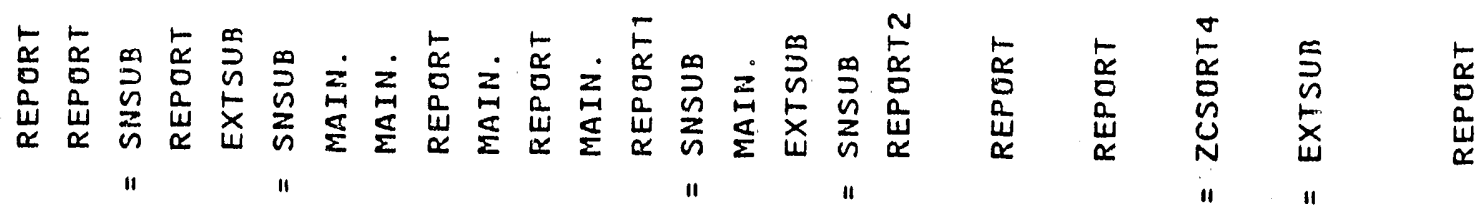

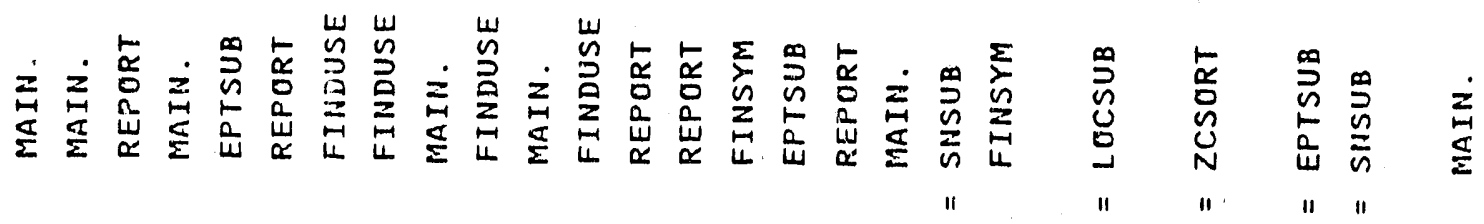

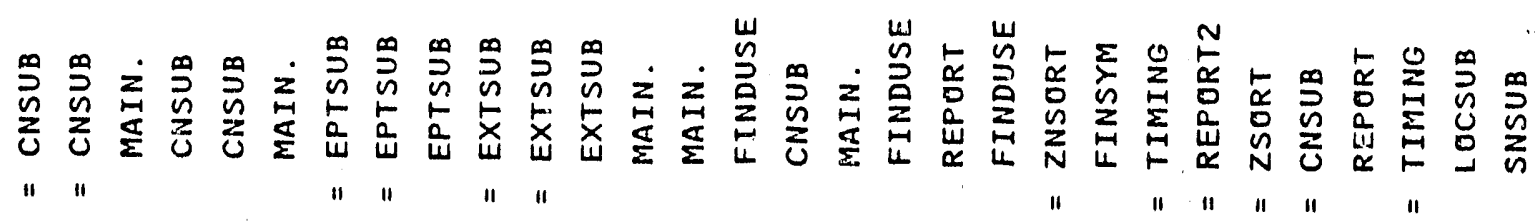

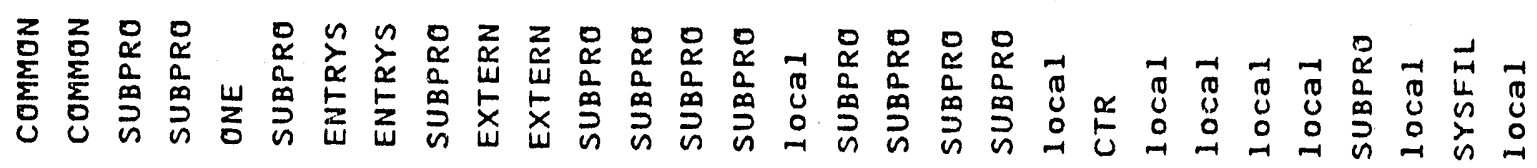

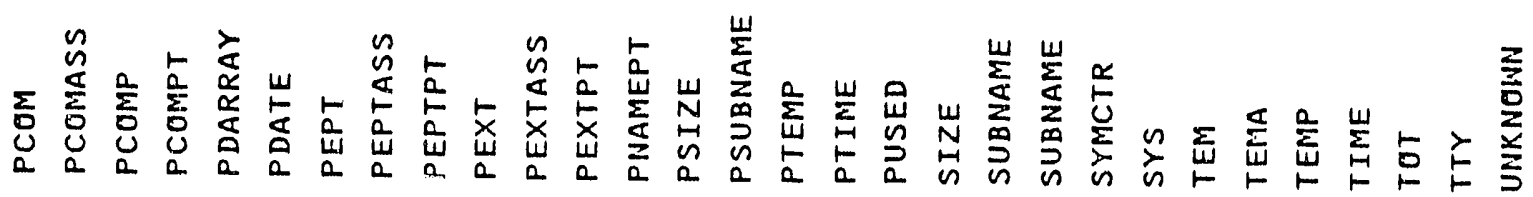


$\sum_{n}^{\infty}$

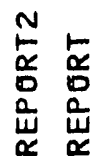

E

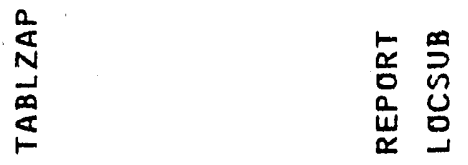

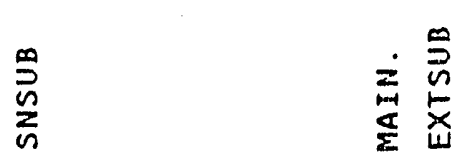

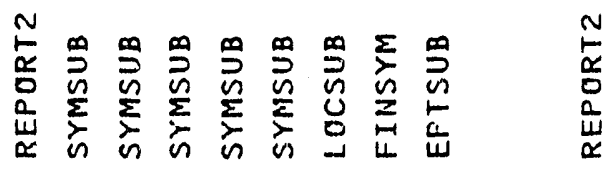

$\frac{m}{m}$

$\stackrel{11}{11}$

- 0

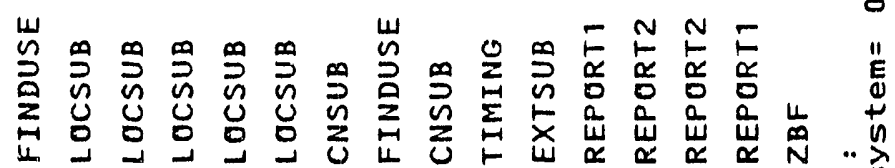

" " " " " " " " " " " "

" " " " "

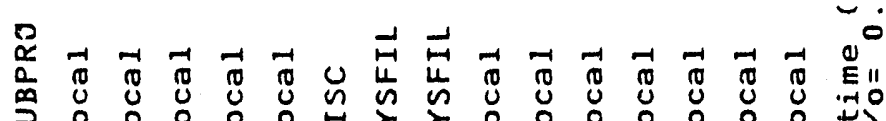

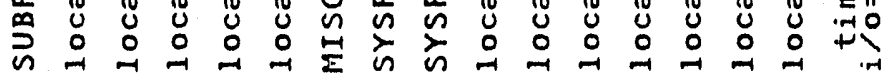

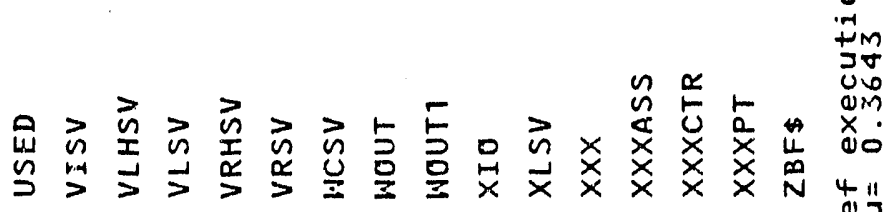

$\begin{array}{rl}4 & 11 \\ 0 & 3 \\ \frac{1}{\times} & 0\end{array}$ 
APPENDDX II 


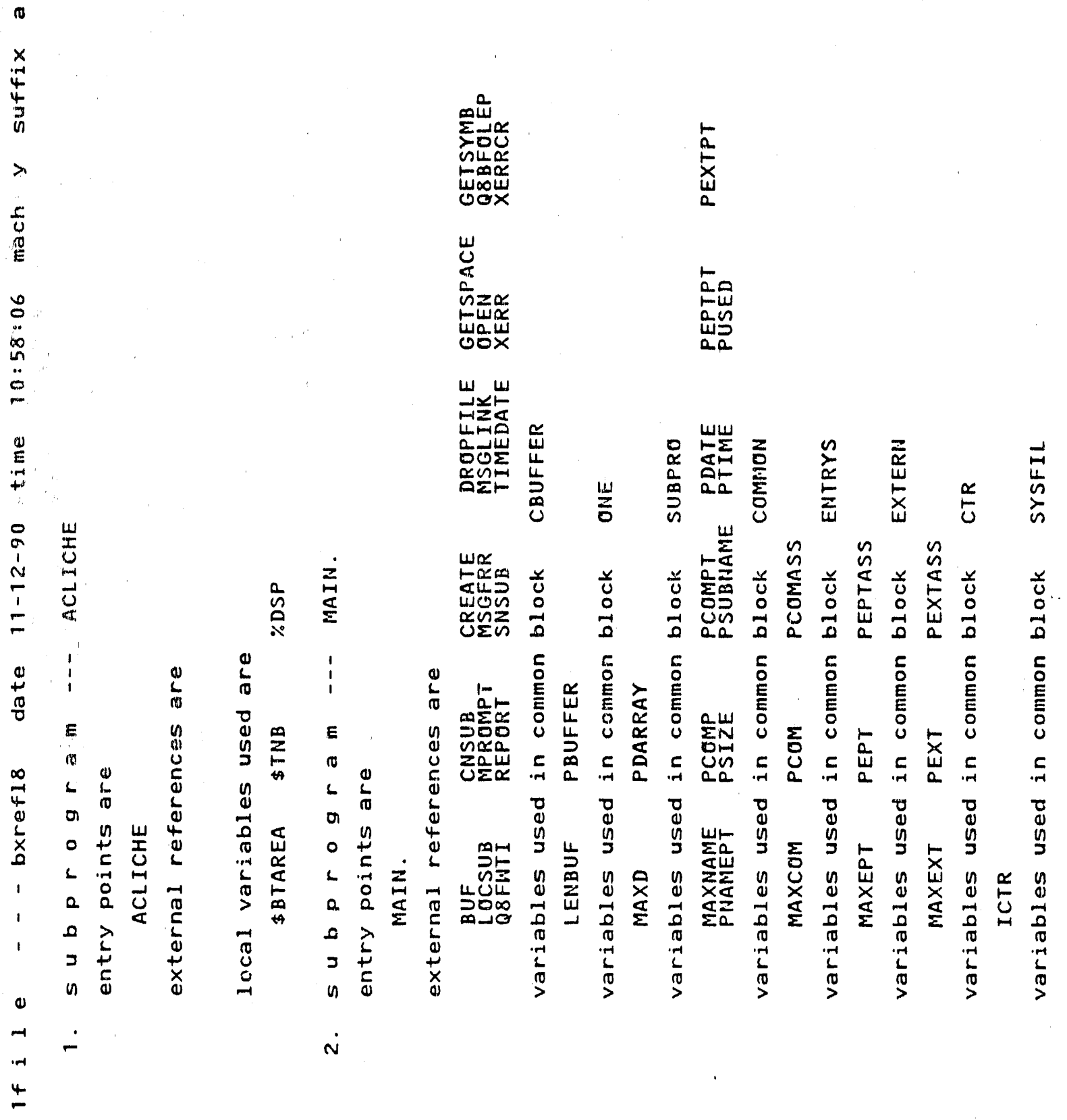



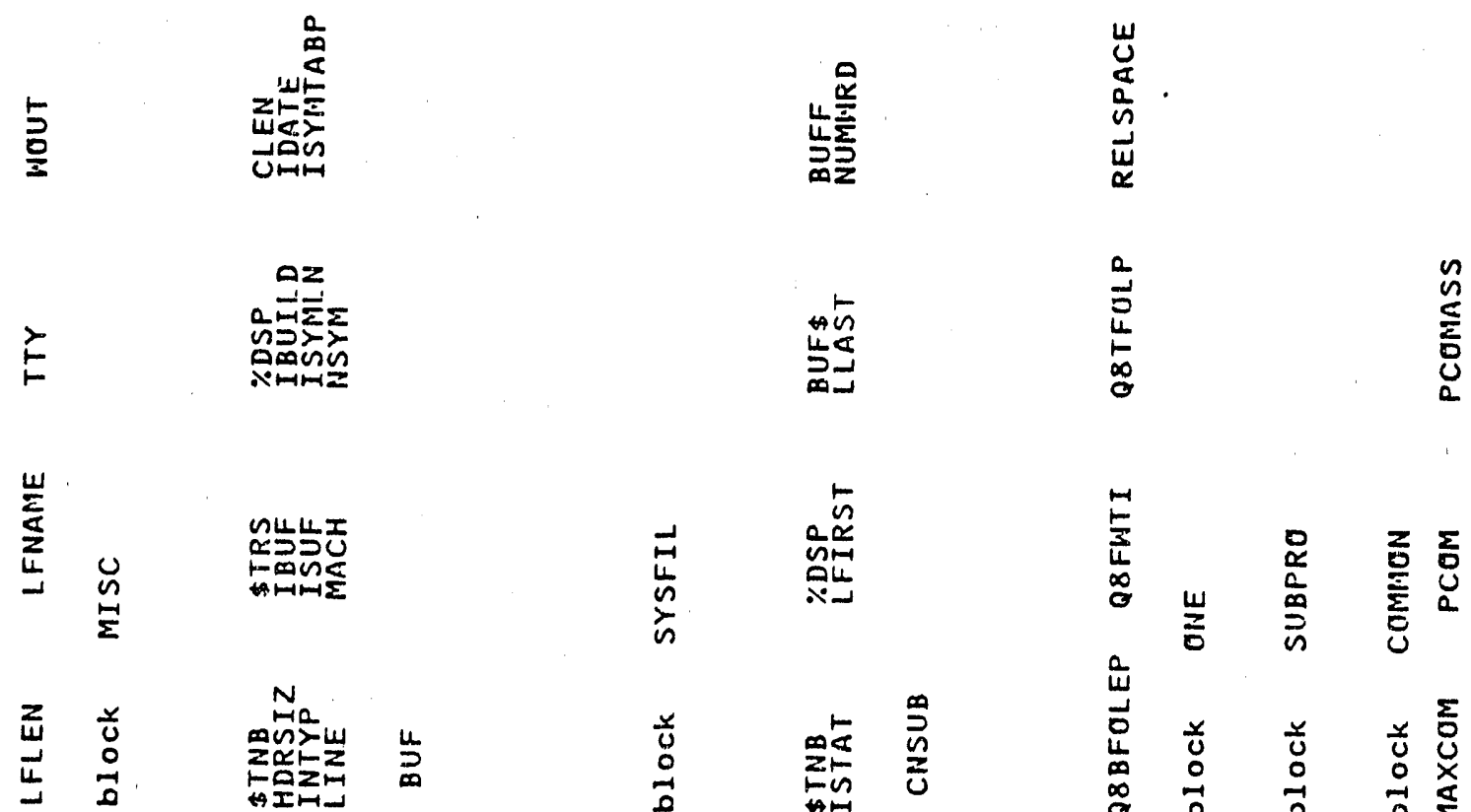

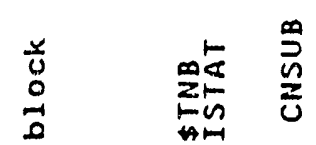

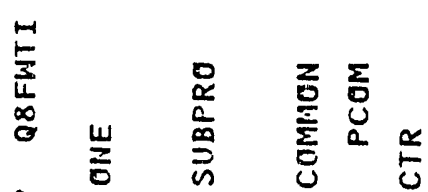

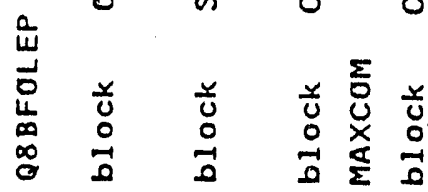

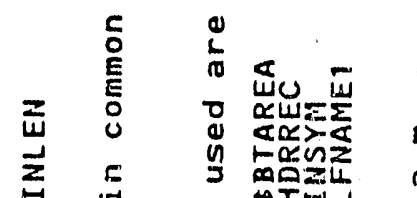

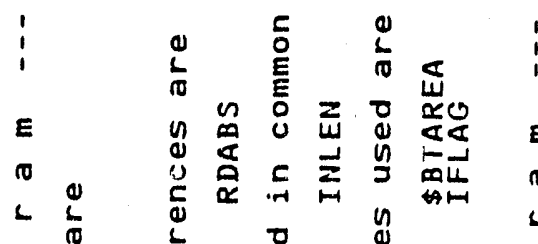

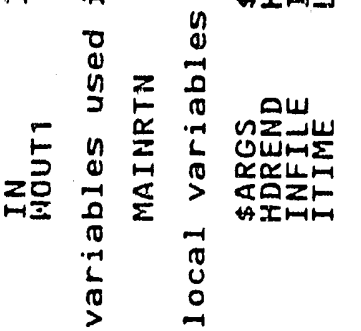

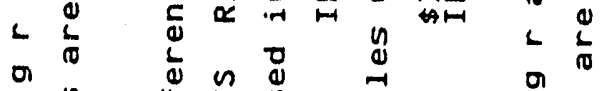

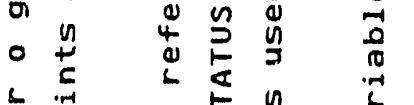

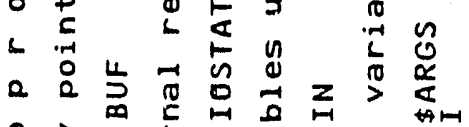

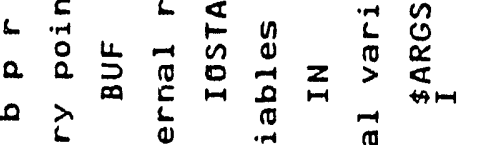

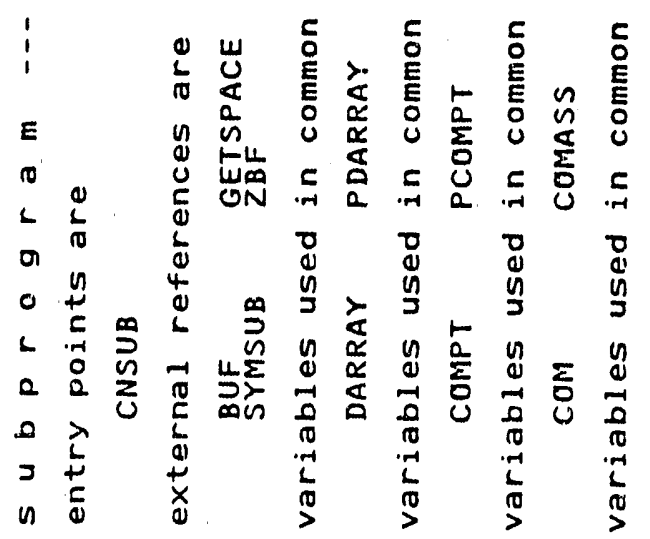




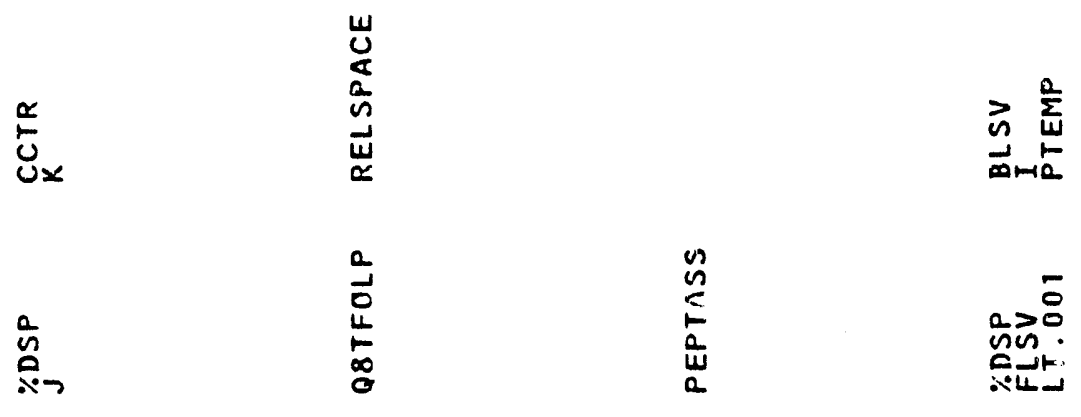

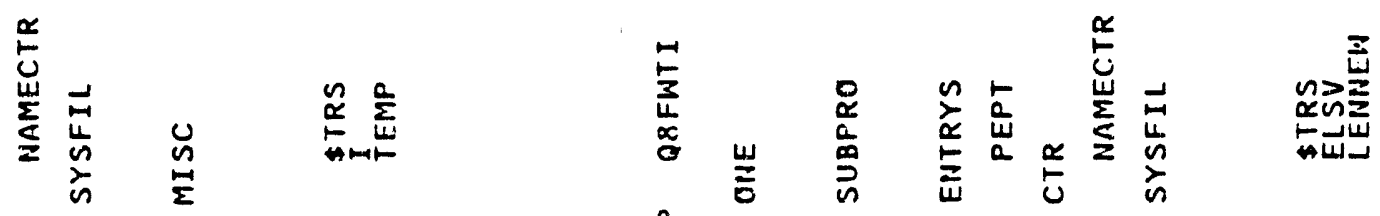

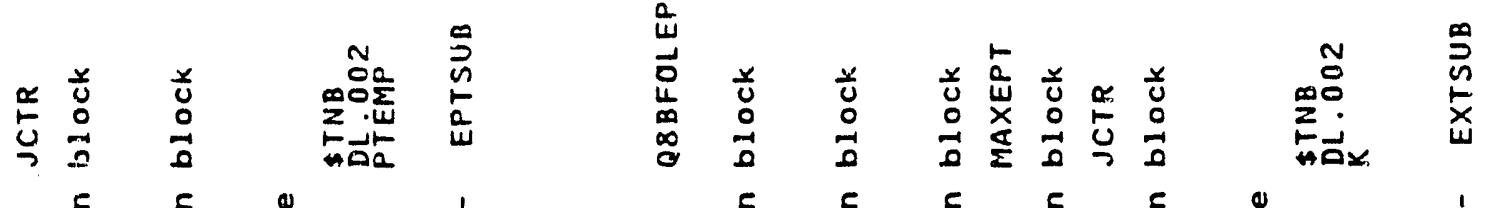

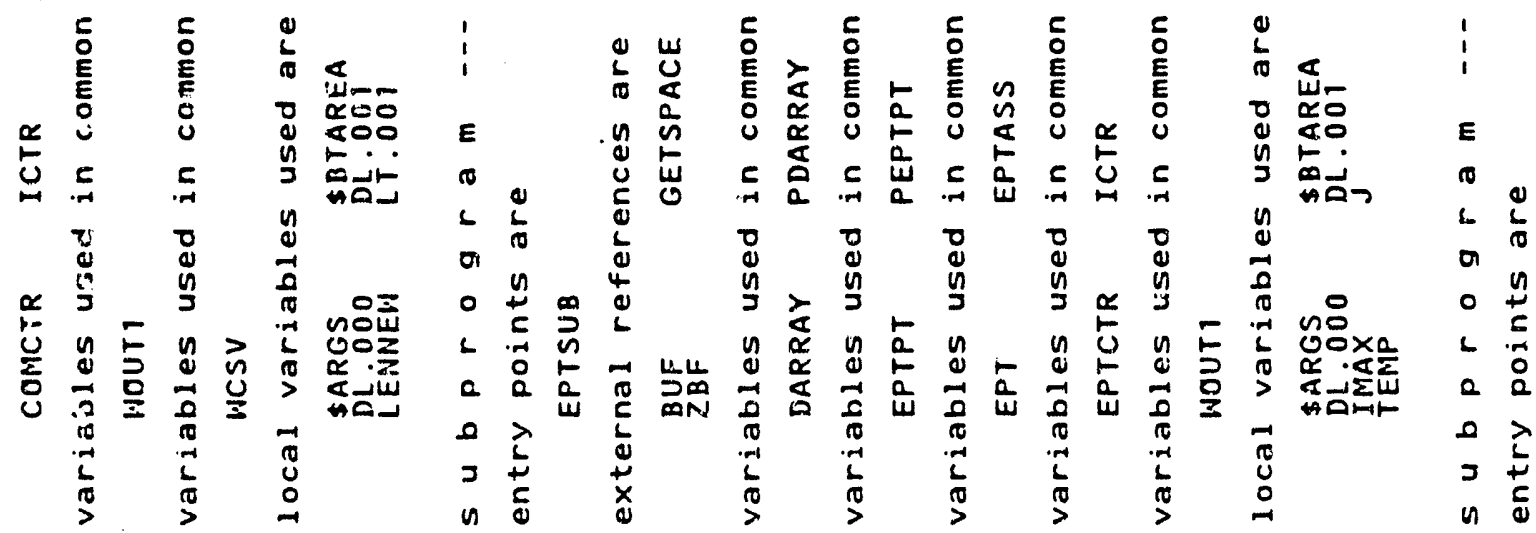

in $\dot{0}$ 


$$
\text { 产 }
$$

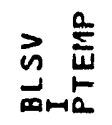

言

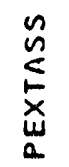

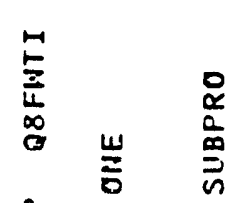

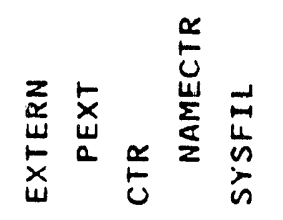

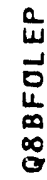

i)

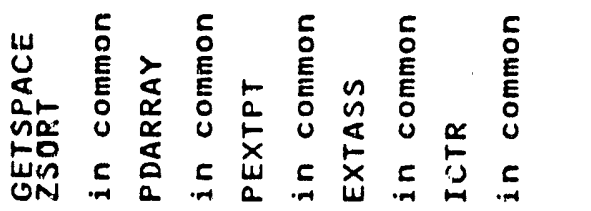

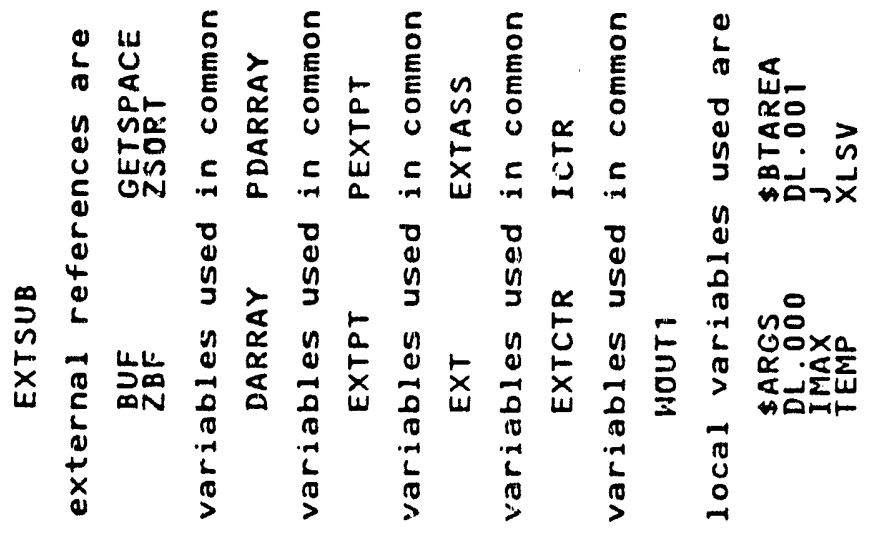

ติ

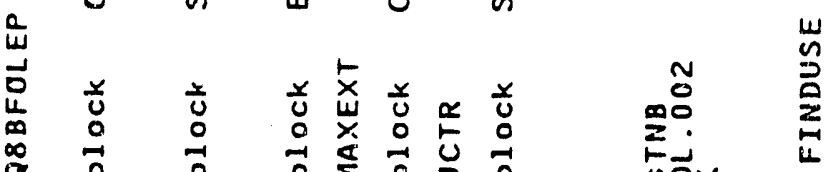

แิ

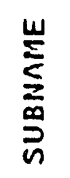

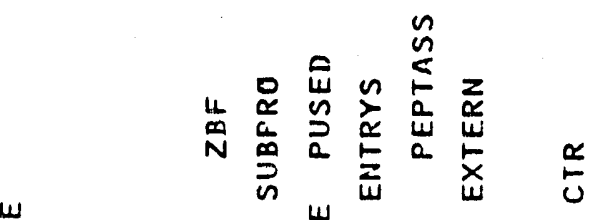

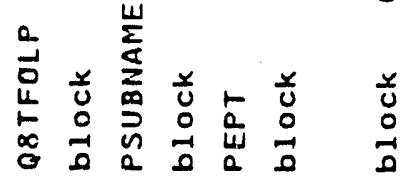

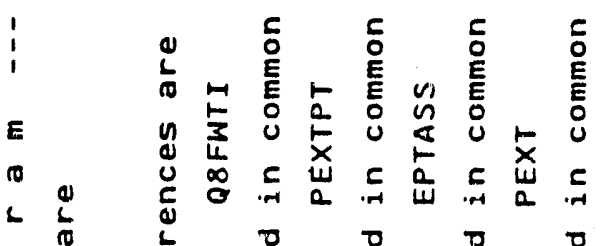

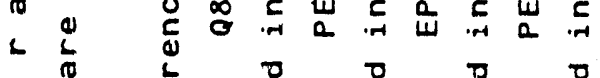

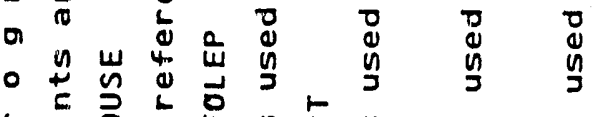

b

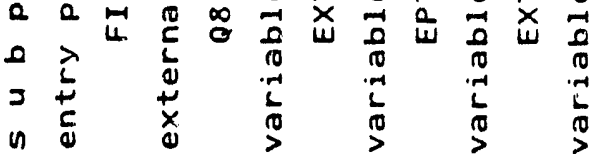




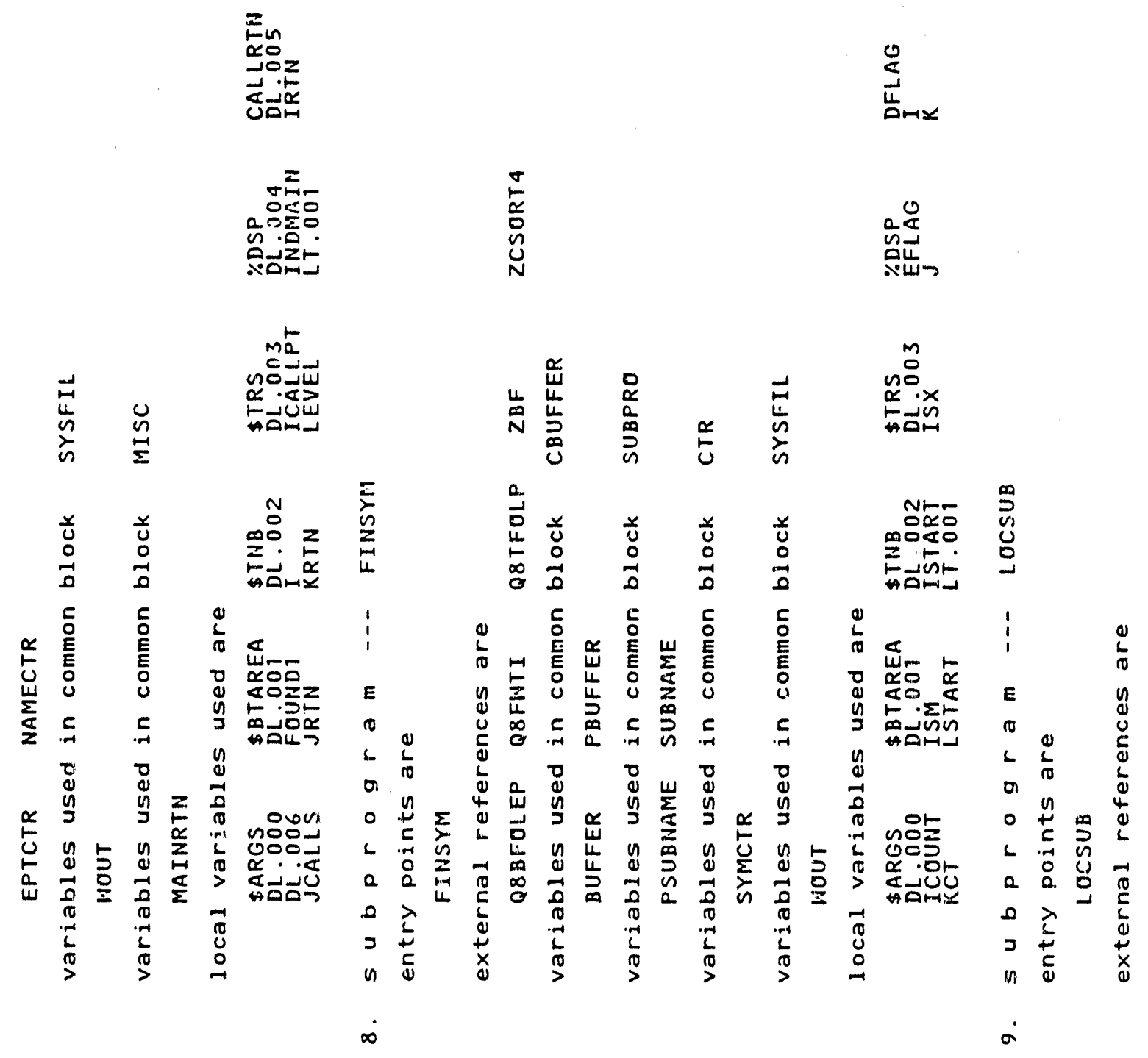


$\frac{a}{0}$ 商敦

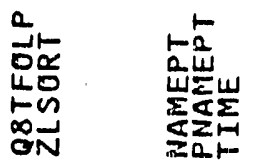

$\underset{\substack{3 \\ \infty \\ 0}}{5}$
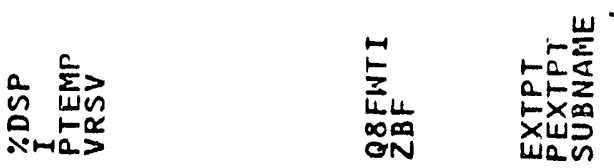

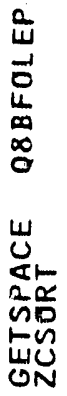

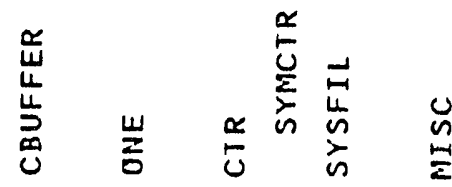

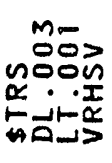

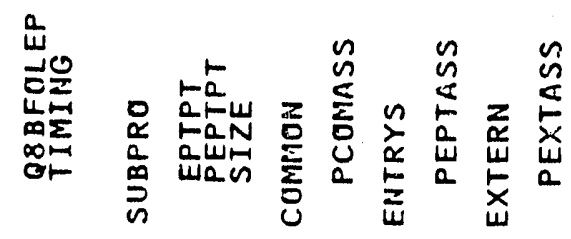

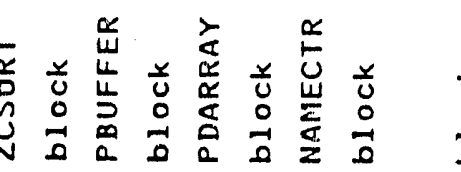

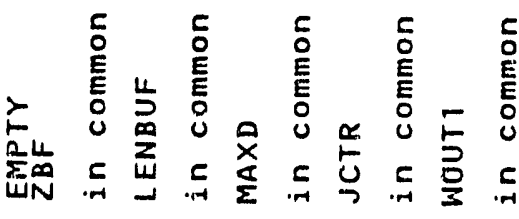

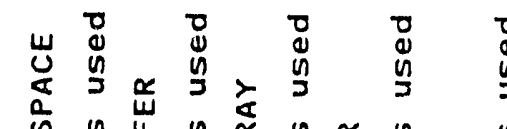
率步

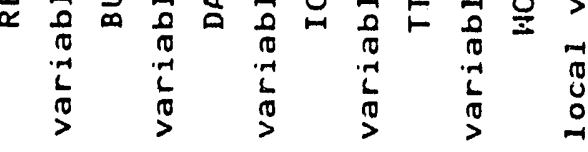

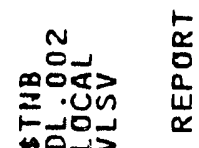

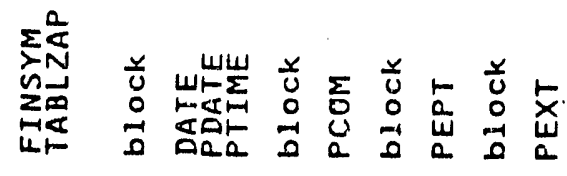

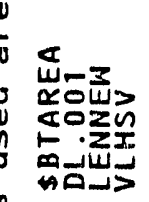

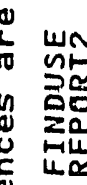

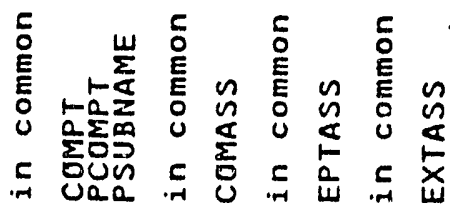

\& $\frac{1}{a}$ L

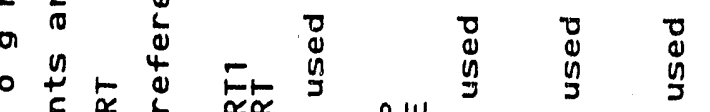

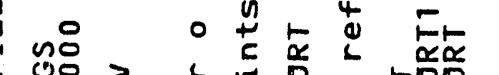

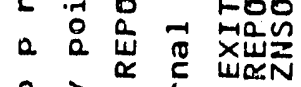

ทேフ

$0 \lambda \ll \frac{5}{6}$ wañ

n 욜

y $\sum$ y $r d y$

@i

n

$\frac{1}{n}$

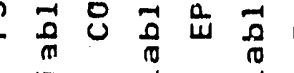

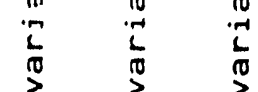

$\therefore$ 


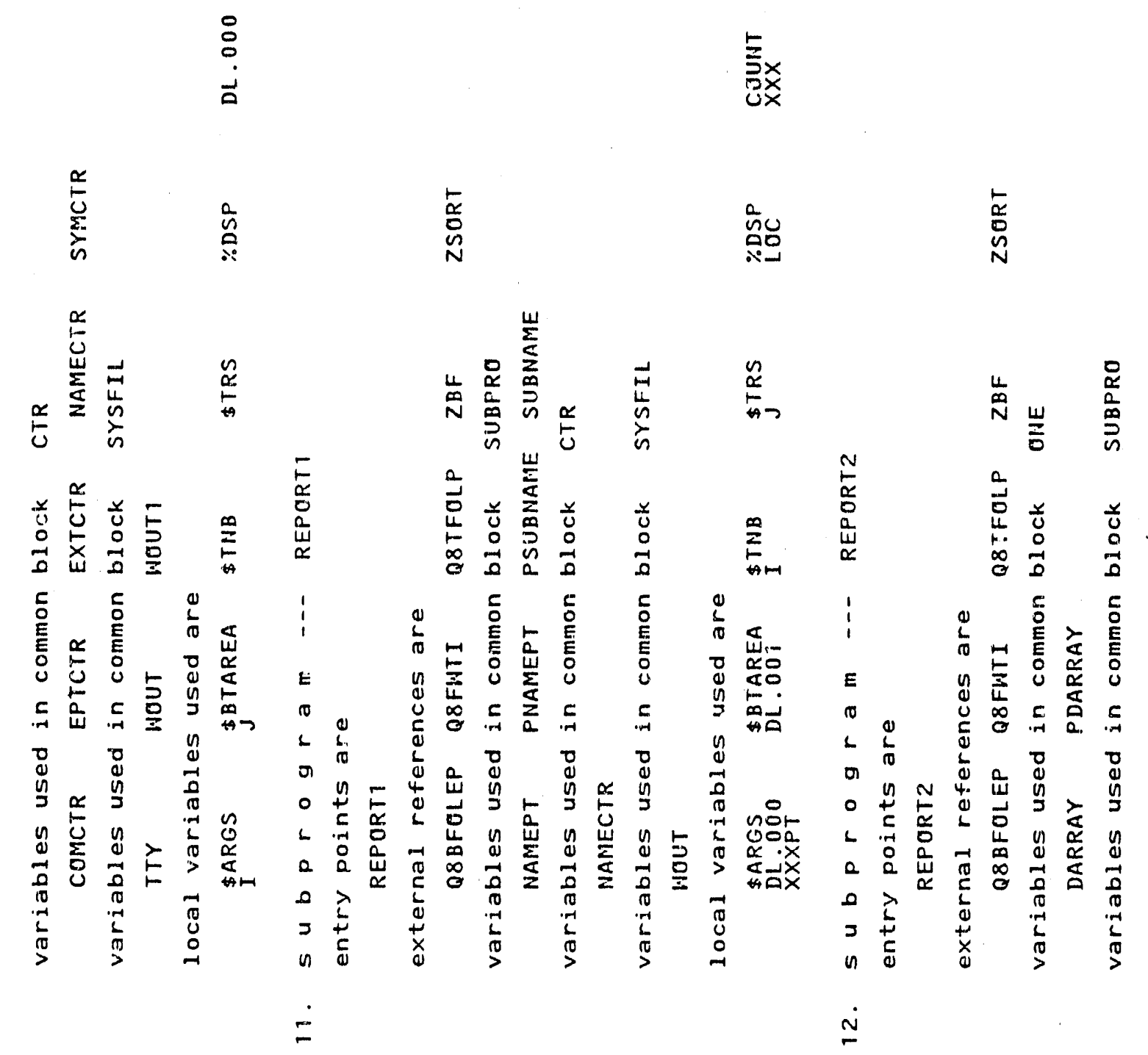




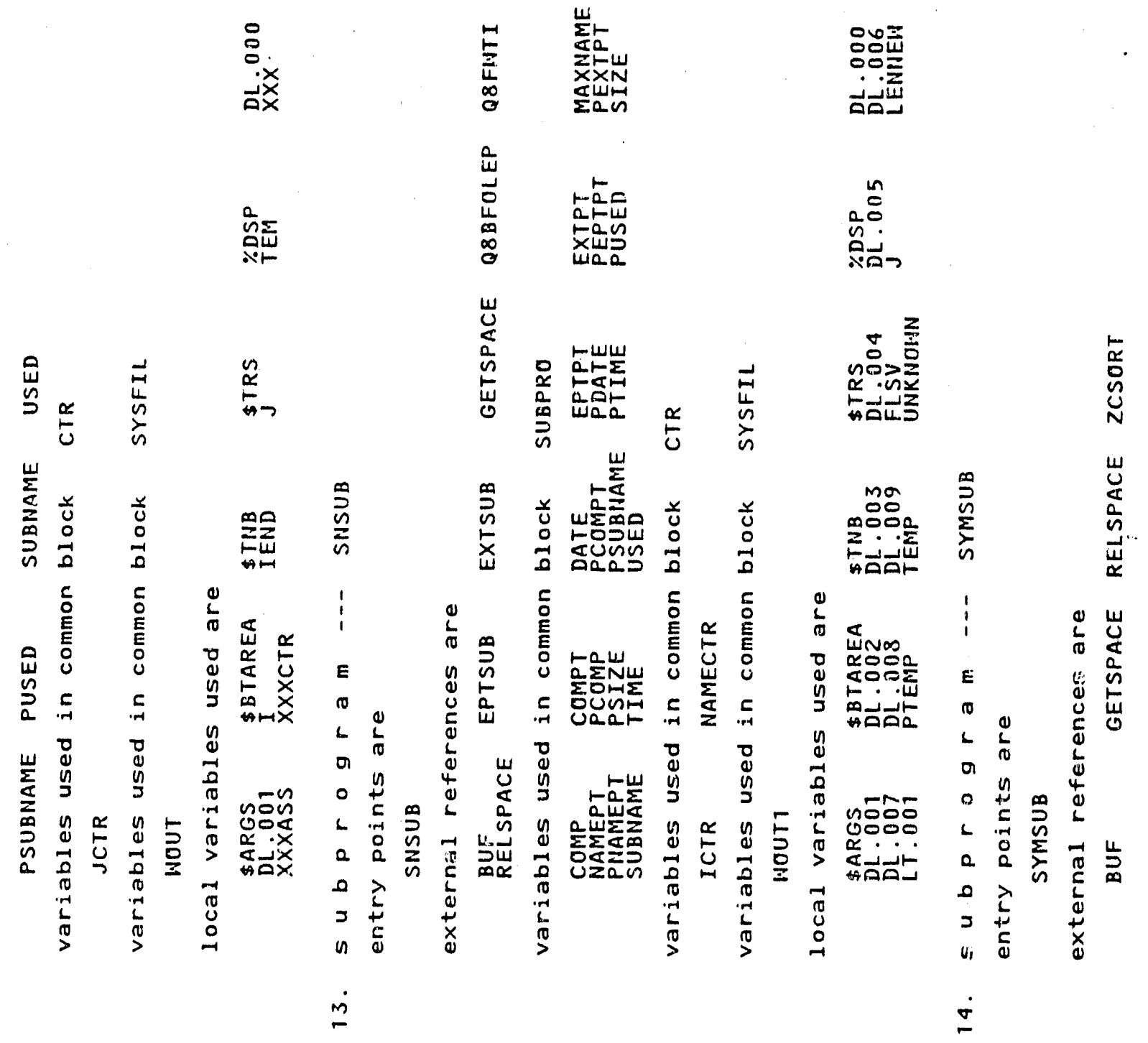




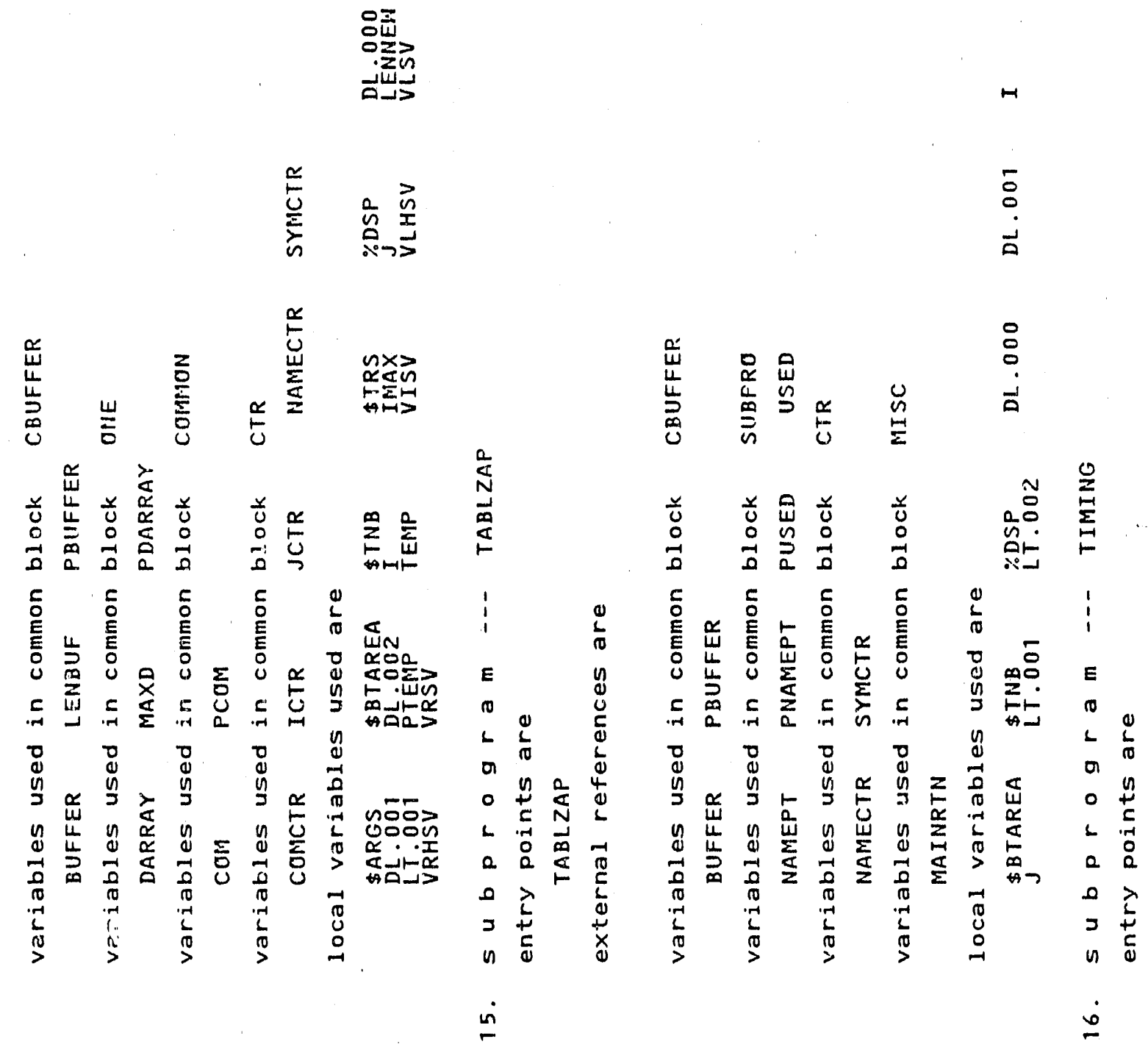




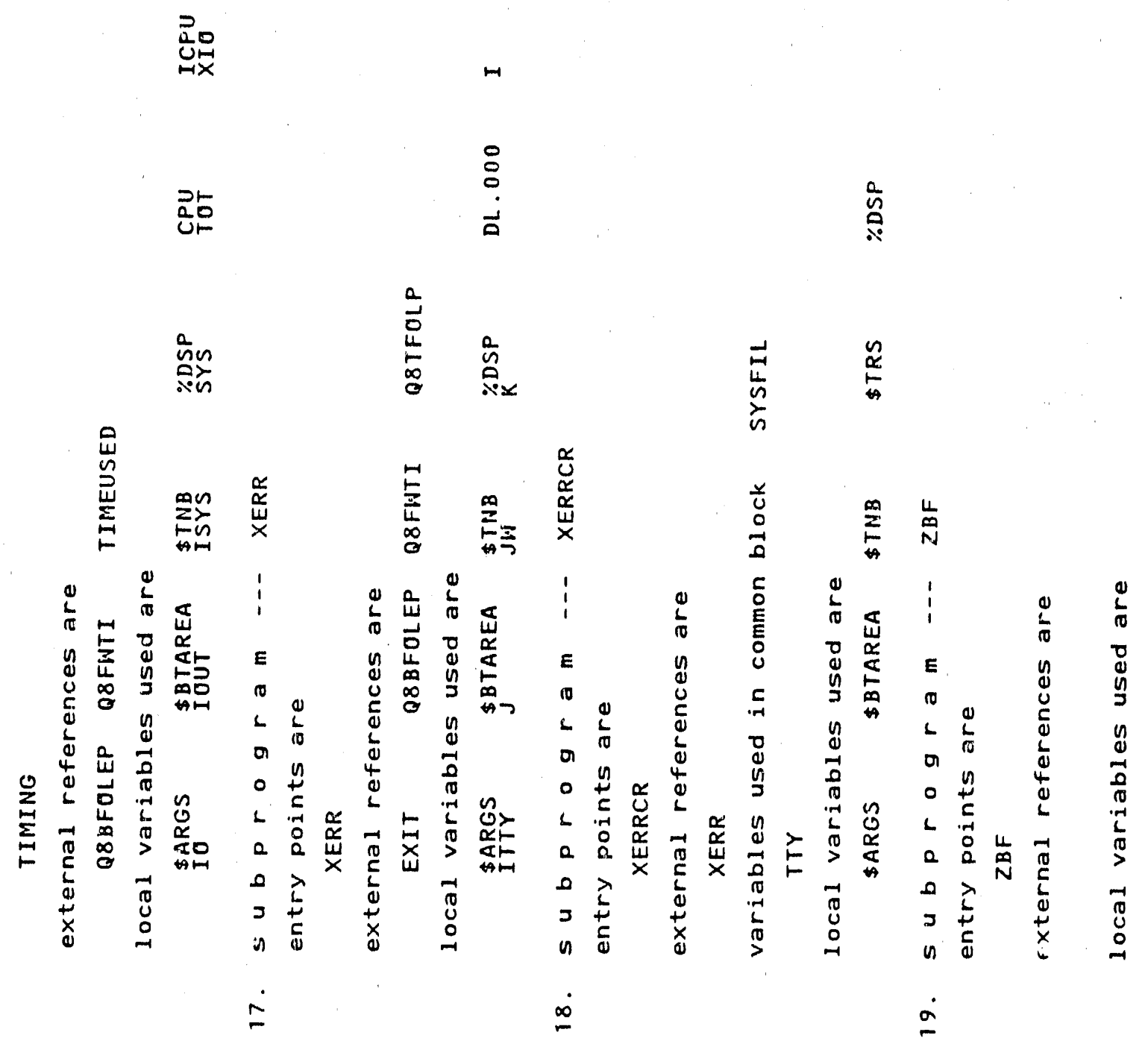




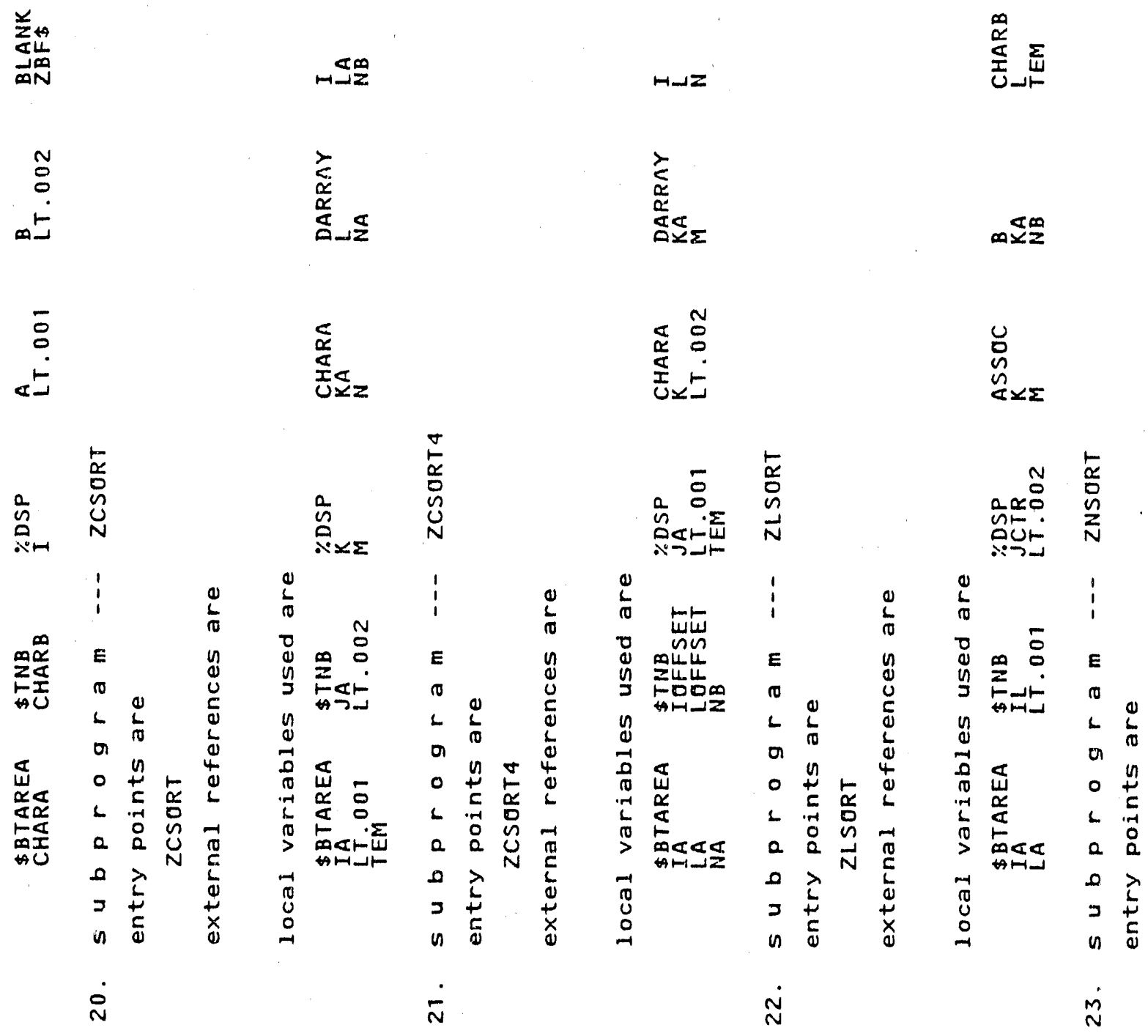



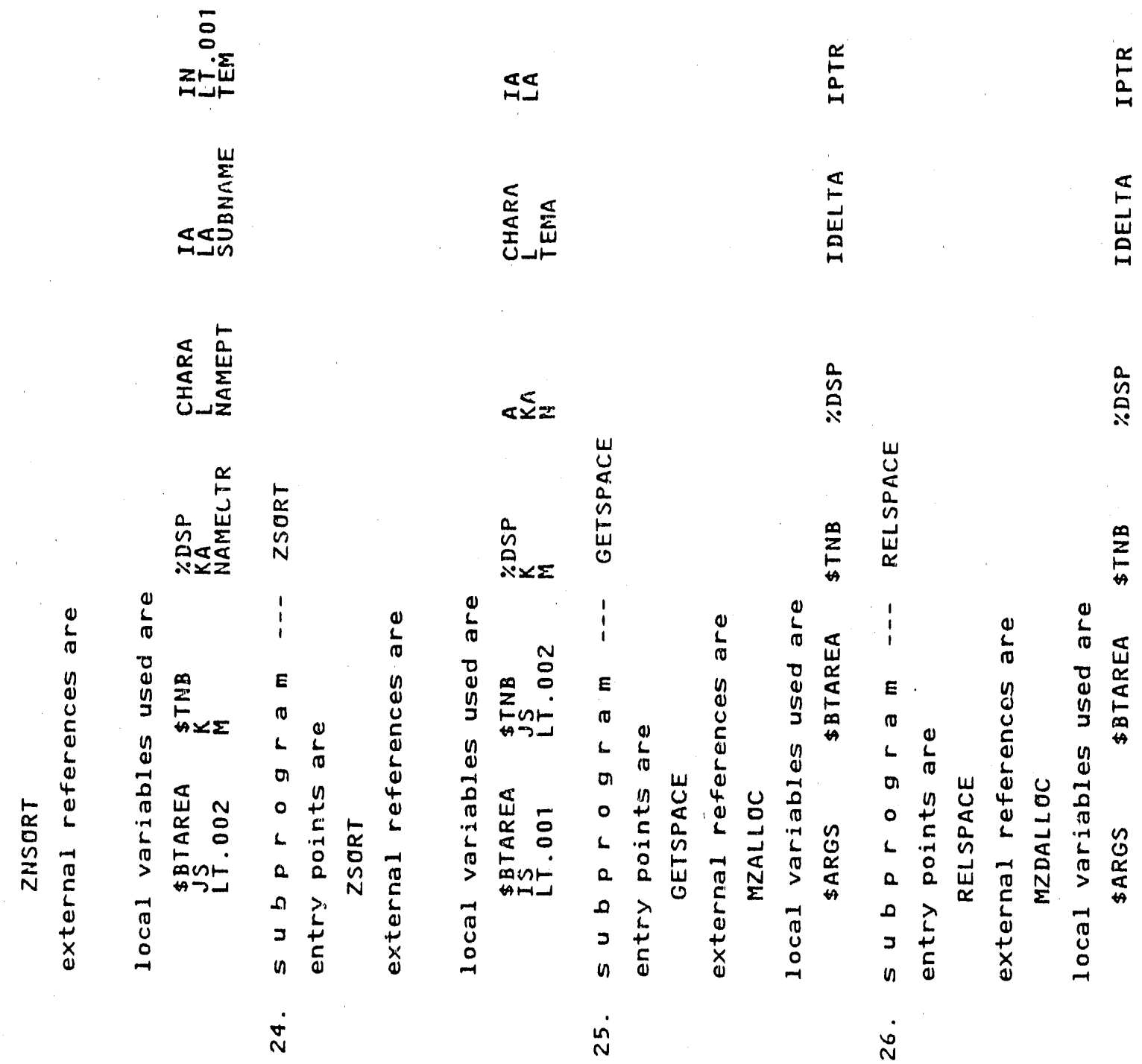


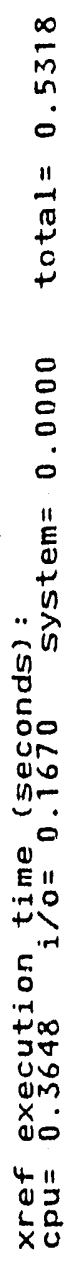



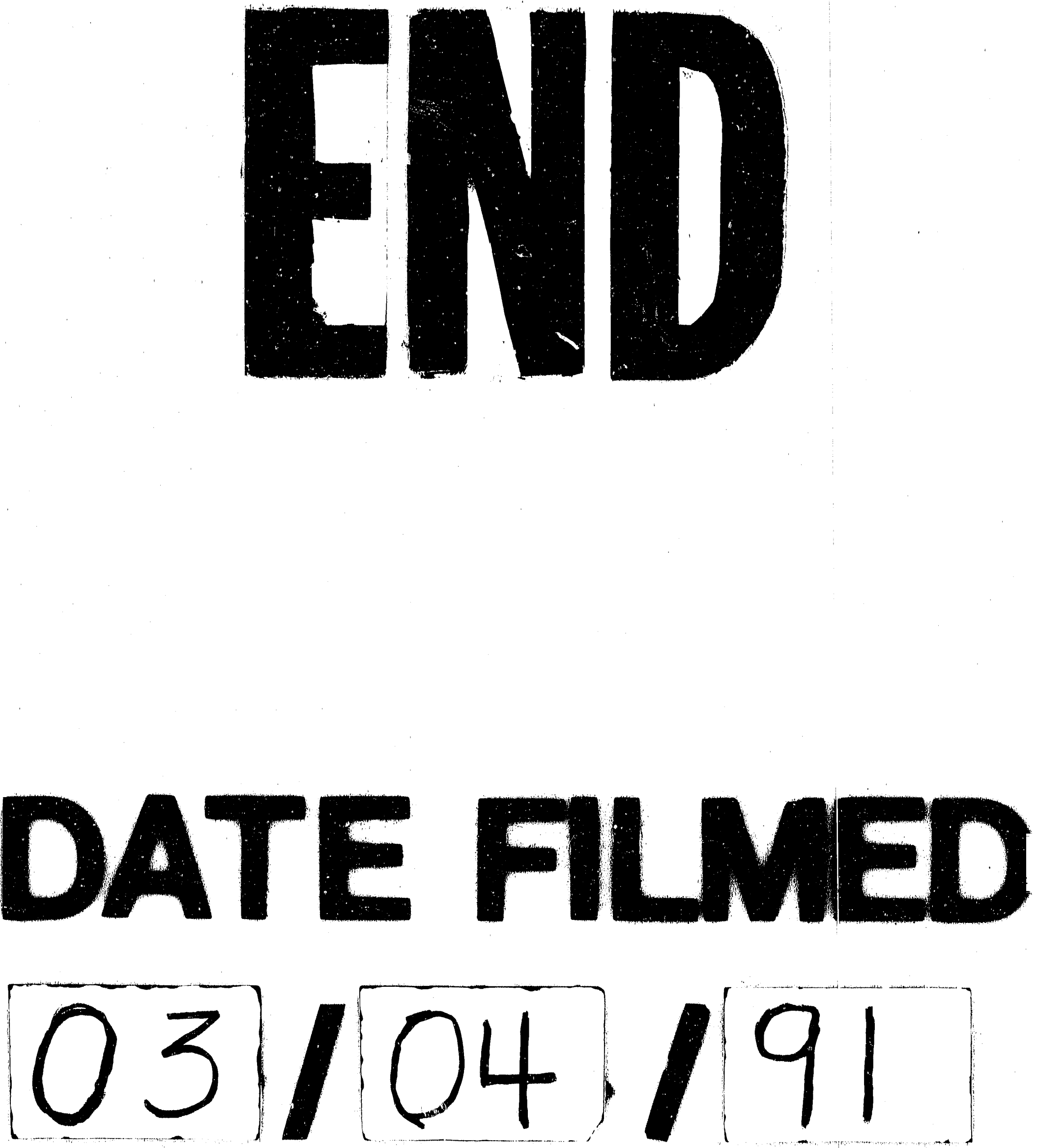
